\title{
SINGULARITIES OF MAXWELL INTERFACE PROBLEMS
}

\author{
Martin Costabel ${ }^{1}$, Monique Dauge ${ }^{1}$ and Serge Nicaise ${ }^{2}$
}

\begin{abstract}
We investigate time harmonic Maxwell equations in heterogeneous media, where the permeability $\mu$ and the permittivity $\varepsilon$ are piecewise constant. The associated boundary value problem can be interpreted as a transmission problem. In a very natural way the interfaces can have edges and corners. We give a detailed description of the edge and corner singularities of the electromagnetic fields.
\end{abstract}

AMS Subject Classification. 35Q60, 35R05, 78A25.

Received: June 5, 1998. Revised: September 1, 1998.

\section{INTRODUCTION}

Physical objects interacting with electromagnetic waves not only tend to have corners and edges, but are frequently composed of several materials with different electric and magnetic properties. The electromagnetic fields then have singularities not only at the exterior corners and edges, but also at the singular points of the interfaces between the different materials.

We show how these singularities can be analyzed using the classical Kondrat'ev method [13]. In reference [8], we studied the singularities at corners and edges of a homogeneous material. Here we continue this investigation of the singularities of solutions of the time-harmonic Maxwell equations by studying the case of piecewise constant coefficients $\varepsilon$ (electric permittivity) and $\mu$ (magnetic permeability). For the case of two materials separated by a plane, see also [5].

We try to describe as explicitly as possible the principal parts of all singular functions of the electric and magnetic fields. We show that all the singular functions can be obtained from those of associated transmission problems for the scalar Laplace operator. Thus one can benefit from the many results that are available on this subject; see $[10,15,16,19]$.

In the case of a homogeneous body [8], the singular functions are generated by those of the Dirichlet and Neumann boundary value problems for the Laplacian. In our heterogeneous case, we also have to consider two problems for the Laplacian. They correspond to the equations for the electrostatic and the magnetostatic potentials. The electrostatic problem is an interface problem for the Laplace operator with exterior Dirichlet boundary conditions and jumps of the normal derivatives at the interfaces determined by the discontinuities of the coefficient $\varepsilon$ (operator $\Delta_{\varepsilon}^{\text {Dir }}$, see (1.7) and Notation (3.3)). For the magnetostatic problem, we have to consider the operator $\Delta_{\mu}^{\mathrm{Neu}}$ (see (1.8) and Notation (3.3)) with Neumann boundary conditions and jumps determined by the discontinuities of the coefficient $\mu$.

Keywords and phrases. Corner singularities, interface problems, transmission conditions, Maxwell equations, electromagnetism.

1 IRMAR, Université de Rennes I, campus de Beaulieu, 35042 Rennes Cedex, France. e-mail: dauge@univ-rennes1.fr

${ }^{2}$ Université de Valenciennes et du Hainaut Cambrésis, LIMAV, B.P. 311, 59304 Valenciennes Cedex, France.

(C) EDP Sciences, SMAI 1999 
As in the homogeneous case [8], we find three types of singularities (type 1,2 and 3). There may be strong singularities that are not even in $H^{1}$. We show that these are of type 1, i.e. gradients of singular functions of the corresponding static problems.

For the singular functions of type 2, there is a difference to the homogeneous case: in [8], we obtained an explicit formula (a differential operator, see Lem. 7.5 of [8]) that gives the Maxwell singularity in terms of the singularity of the opposite static potential problem. In our heterogeneous case, the exponent of the singularity is still equal to an exponent of the opposite static potential problem. For the angular part of the singular function, however, we find an additional term, see (5.3), that involves the solution of an inhomogeneous scalar interface problem. Thus the type 2 singularities of the electric fields have the same exponents as the magnetostatic potentials, but their angular parts contain a term corresponding to an electrostatic field generated by interface charges depending on the jumps $[\varepsilon \mu]$ of the index of refraction.

Another important difference to the homogeneous case is that the regularity for the interface problems can be much lower, even with regular data. Thus, in the homogeneous case, one has at least $H^{1 / 2}$ regularity for Lipschitz domains [6] and $H^{1}$ regularity for convex domains [20]. Here, we find only 0 as a limit for the regularity. Thus for any $s>0$ there are examples where the solution is not in $H^{s}$. If there are only two materials the lower limit of regularity is $1 / 4$ for arbitrary polyhedra and $1 / 2$ for convex domains.

For the two-dimensional case (which governs also the edge singularities in dimension 3), one has simple formulas in the homogeneous case: they show that the strongest singularity is of type 1 and that the lower limit of regularity is $\pi / \omega$ if $\omega \in(0,2 \pi)$ is the largest opening angle. This holds for both the electric and the magnetic field.

In the heterogeneous case, due to the different behaviors of the coefficients $\varepsilon$ and $\mu$, the electric and magnetic fields will have, in general, different regularities. As usual their regularity is limited by the leading singularity. If this leading singularity is of type 1 , the regularity is $s-1$, with $s$ the regularity of the corresponding static problem. If not, the leading singularity is of type 2 , and the regularity is the same as the regularity of the opposite static problem. In the two-dimensional homogeneous case, the second possibility never happens, while in the heterogeneous case, there are cases where the leading singularity is not of type 1 , but of type 2 .

Let us give an example. In a typical case of several dielectric materials (three are sufficient) with strongly varying $\varepsilon$, but constant $\mu$, in a convex polygon with largest opening $\omega$, one has $H^{2+\gamma}$ regularity for the magnetostatic potential, with $\gamma>0$ any number $<\pi / \omega-1$. For the electrostatic potential one may have only $H^{1+\delta}$ regularity with any $\delta>0$. Thus the type 1 singularity for the magnetic field has regularity $H^{1+\gamma}$, compared to the $H^{1+\delta}$ regularity for the type 2 singularity. It is easy to have $\delta<\gamma$ (take three adjacent sectors of opening $\pi / 4$ and $\varepsilon$ equal to 1 in the exterior sectors and to 100 in the middle sector: then $\gamma=0.3333$ and $\delta=0.1793)$. In such a situation, the electric field has only $H^{\delta}$ regularity (type 1 ) while the magnetic field has $H^{1+\delta}$ regularity. Such a difference of 1 between these two regularities is the maximum possible. (See also Rem. (8.2) for an example where $\gamma=+\infty$ and $\delta$ is close to 0 .)

In Section 1, we recall the regularized variational formulation of Maxwell's equations for heterogeneous materials. We define the two associated scalar potential operators $\Delta_{\varepsilon}^{\text {Dir }}$ and $\Delta_{\mu}^{\text {Neu }}$.

In Section 2, we characterize the closure of the subspace of smooth functions in the natural variational spaces associated with the electric and magnetic fields.

In Section 3, we give two different decompositions of the variational spaces. In the first case, the regular part is in $H^{1}$ on the whole domain, thus has no jumps across the interfaces, whereas in the second case, the regular part has jumps in the components normal to the interfaces. In both cases, the singular parts are gradients.

In Section 4, we state the necessary results on scalar interface problems for the Laplacian. In Section 5 , the three types of Maxwell corner singularities and in Section 6, the edge singularities are studied.

Section 7 gives some conclusions about $H^{s}$ regularity in general and in several particular cases. We give in Section 8 proofs for the results about minimal edge regularity for the Laplace interface problems on which the Maxwell regularity results are based.

We shall use the following geometric and analytic setting: we assume that $\Omega$ is a Lipschitz polyhedron, which means that $\Omega$ is a bounded Lipschitz domain with piecewise plane boundary. We also assume that $\varepsilon$ and $\mu$ are 
piecewise constant $>0$ on $\Omega$, determining a partition $\mathscr{P}$ of $\Omega$ in a finite set of Lipschitz polyhedra $\Omega_{1}, \ldots, \Omega_{J}$ : on each $\Omega_{j}, \varepsilon=\varepsilon_{j}$ and $\mu=\mu_{j}$ with $\varepsilon_{j}$ and $\mu_{j}$ positive constants. We denote by $F_{j k}$ the (open) faces of $\Omega_{j}$. Let $\mathscr{F}_{\text {int }}$ be the set of the interior faces (contained in $\Omega$ ) and $\mathscr{F}_{\text {ext }}$ the set of the exterior faces (contained in $\partial \Omega$ ).

In general, we will denote by bold letters the functional spaces for the fields. Thus $H^{s}(\Omega)$ denotes the usual Sobolev space on $\Omega$ and $\boldsymbol{H}^{s}(\Omega)$ denotes $H^{s}(\Omega)^{3}$. We also need for $s \geq 1 / 2$ piecewise $H^{s}$ functions relative to the partition $\mathscr{P}$

$$
P H^{s}(\Omega, \mathscr{P})=\left\{\varphi \in L^{2}(\Omega) \mid \quad \varphi_{j} \in H^{s}\left(\Omega_{j}\right), \quad j=1, \ldots, J\right\} .
$$

Here, of course, $\varphi_{j}$ denotes the restriction of $\varphi$ to $\Omega_{j}$. For the fields we set

$$
P \boldsymbol{H}^{s}(\Omega, \mathscr{P})=P H^{s}(\Omega, \mathscr{P})^{3} .
$$

We will also denote by $P H^{1 / 2}\left(\mathscr{F}_{\text {int }}\right)$ the product of the spaces $H^{1 / 2}(F)$ for $F \in \mathscr{F}_{\text {int }}$ and similarly for $\mathscr{F}_{\text {ext }}$. Finally, as usual for Maxwell equations, we need spaces of fields with square integrable curls:

$$
\boldsymbol{H}(\operatorname{curl} ; \Omega)=\left\{\boldsymbol{u} \in L^{2}(\Omega)^{3} \mid \quad \operatorname{curl} \boldsymbol{u} \in L^{2}(\Omega)^{3}\right\}
$$

and with square integrable divergences (here $\xi=\varepsilon$ or $\mu$ )

$$
\boldsymbol{H}(\operatorname{div} ; \xi ; \Omega)=\left\{\boldsymbol{u} \in L^{2}(\Omega)^{3} \mid \operatorname{div}(\xi \boldsymbol{u}) \in L^{2}(\Omega)\right\}
$$

As usual, if $\xi \equiv 1, \boldsymbol{H}(\operatorname{div} ; \xi ; \Omega)$ is denoted $\boldsymbol{H}(\operatorname{div} ; \Omega)$ for short.

\section{MAXWELl FORMUlations}

Classical time harmonic Maxwell equations are given by

$$
\operatorname{curl} \boldsymbol{E}-i \omega \mu \boldsymbol{H}=0 \quad \text { and } \quad \operatorname{curl} \boldsymbol{H}+i \omega \varepsilon \boldsymbol{E}=\boldsymbol{J} \quad \text { in } \quad \Omega .
$$

Here $\boldsymbol{E}$ is the electric part and $\boldsymbol{H}$ the magnetic part of the electromagnetic field. The right hand side $\boldsymbol{J}$ is the current density. The exterior boundary conditions on $\partial \Omega$ are those of the perfect conductor $(\boldsymbol{n}$ denotes the unit outer normal on $\partial \Omega$ ):

$$
\boldsymbol{E} \times \boldsymbol{n}=0 \quad \text { and } \quad \boldsymbol{H} \cdot \boldsymbol{n}=0 \quad \text { on } \quad \partial \Omega .
$$

The natural variational spaces are $\boldsymbol{X}_{N}(\Omega, \varepsilon)$ for the electric field and $\boldsymbol{X}_{T}(\Omega, \mu)$ for the magnetic field according to

$$
\boldsymbol{X}_{N}(\Omega, \varepsilon)=\{\boldsymbol{u} \in \boldsymbol{H}(\operatorname{curl} ; \Omega) \cap \boldsymbol{H}(\operatorname{div} ; \varepsilon ; \Omega) \mid \quad \boldsymbol{u} \times \boldsymbol{n}=0 \quad \text { on } \quad \partial \Omega\}
$$

and

$$
\boldsymbol{X}_{T}(\Omega, \mu)=\{\boldsymbol{u} \in \boldsymbol{H}(\operatorname{curl} ; \Omega) \cap \boldsymbol{H}(\operatorname{div} ; \mu ; \Omega) \mid \boldsymbol{u} \cdot \boldsymbol{n}=0 \quad \text { on } \quad \partial \Omega\} .
$$

Any field $\boldsymbol{u}$ belonging to one of these spaces is in $\boldsymbol{H}\left(\operatorname{curl} ; \Omega_{j}\right) \cap \boldsymbol{H}\left(\operatorname{div} ; \Omega_{j}\right)$ for each $j$ and satisfies additional jump conditions at the interior interfaces $F \in \mathscr{F}_{\text {int }}$ :

$$
\begin{aligned}
& \boldsymbol{X}_{N}(\Omega, \varepsilon)=\left\{\boldsymbol{u} \in L^{2}(\Omega)^{3} \mid \quad \operatorname{curl} \boldsymbol{u}_{j} \in L^{2}\left(\Omega_{j}\right)^{3}, \quad \operatorname{div} \boldsymbol{u}_{j} \in L^{2}\left(\Omega_{j}\right),\right. \\
& {[\boldsymbol{u} \times \boldsymbol{n}]_{F}=0, \quad[\varepsilon \boldsymbol{u} \cdot \boldsymbol{n}]_{F}=0, \quad \forall F \in \mathscr{F}_{\text {int }}} \\
& \left.\boldsymbol{u} \times\left.\boldsymbol{n}\right|_{F}=0, \quad \forall F \in \mathscr{F}_{\text {ext }}\right\}
\end{aligned}
$$


and

$$
\begin{aligned}
& \boldsymbol{X}_{T}(\Omega, \mu)=\left\{\boldsymbol{u} \in L^{2}(\Omega)^{3} \mid \quad \operatorname{curl} \boldsymbol{u}_{j} \in L^{2}\left(\Omega_{j}\right)^{3}, \quad \operatorname{div} \boldsymbol{u}_{j} \in L^{2}\left(\Omega_{j}\right),\right. \\
& {[\boldsymbol{u} \times \boldsymbol{n}]_{F}=0, \quad[\mu \boldsymbol{u} \cdot \boldsymbol{n}]_{F}=0, \quad \forall F \in \mathscr{F}_{\text {int }}} \\
& \left.\left.\boldsymbol{u} \cdot \boldsymbol{n}\right|_{F}=0, \quad \forall F \in \mathscr{F}_{\text {ext }}\right\}
\end{aligned}
$$

where the jump $[\boldsymbol{v} \times \boldsymbol{n}]_{F}$ is equal to $\left.\left(\boldsymbol{v}_{j} \times \boldsymbol{n}_{j}-\boldsymbol{v}_{j^{\prime}} \times \boldsymbol{n}_{j}\right)\right|_{F}$ if $F$ belongs to $\partial \Omega_{j}$ and to $\partial \Omega_{j^{\prime}}$, with $\boldsymbol{v}_{j}$ the restriction of $\boldsymbol{v}$ to $\Omega_{j}$ and with $\boldsymbol{n}_{j}$ the exterior unit normal to $\partial \Omega_{j}$.

We can formulate elliptic variational problems either for $\boldsymbol{E}$ or $\boldsymbol{H}$. We introduce the following two formulations:

$$
\begin{aligned}
\boldsymbol{u} \in \boldsymbol{X}_{N}(\Omega, \varepsilon), \quad \forall \boldsymbol{v} \in \boldsymbol{X}_{N}(\Omega, \varepsilon), \\
\int_{\Omega} \mu^{-1} \operatorname{curl} \boldsymbol{u} \cdot \operatorname{curl} \boldsymbol{v}+\operatorname{div} \varepsilon \boldsymbol{u} \operatorname{div} \varepsilon \boldsymbol{v}-\omega^{2} \varepsilon \boldsymbol{u} \cdot \boldsymbol{v}=\langle\boldsymbol{f}, \boldsymbol{v}\rangle,
\end{aligned}
$$

where $\langle\boldsymbol{f}, \boldsymbol{v}\rangle=i \omega\langle\boldsymbol{J}, \boldsymbol{v}\rangle+\frac{1}{i \omega}\langle\operatorname{div} \boldsymbol{J}, \operatorname{div} \varepsilon \boldsymbol{v}\rangle$, and

$$
\begin{aligned}
\boldsymbol{u} \in \boldsymbol{X}_{T}(\Omega, \mu), \quad \forall \boldsymbol{v} \in \boldsymbol{X}_{T}(\Omega, \mu), \\
\int_{\Omega} \varepsilon^{-1} \operatorname{curl} \boldsymbol{u} \cdot \operatorname{curl} \boldsymbol{v}+\operatorname{div} \mu \boldsymbol{u} \operatorname{div} \mu \boldsymbol{v}-\omega^{2} \mu \boldsymbol{u} \cdot \boldsymbol{v}=\langle\boldsymbol{h}, \boldsymbol{v}\rangle,
\end{aligned}
$$

where $\langle\boldsymbol{h}, \boldsymbol{v}\rangle=\left\langle\varepsilon^{-1} \boldsymbol{J}, \operatorname{curl} \boldsymbol{v}\right\rangle$. If $(\boldsymbol{E}, \boldsymbol{H})$ solves the Maxwell equations (1.1-1.2), then $\boldsymbol{E}$ is solution of (1.5) and $\boldsymbol{H}$ of (1.6). The converse also holds, see [8], if $\omega^{2}$ does not belong to the spectrum of the operators $-\Delta_{\varepsilon}^{\text {Dir }}$ and $-\Delta_{\mu}^{\text {Neu }}$ naturally associated with equations (1.1):

- $-\Delta_{\varepsilon}^{\text {Dir }}$ is defined from $\stackrel{\circ}{H^{1}}(\Omega)$ into its dual $H^{-1}(\Omega)$ by

$$
\forall \Phi, \Psi \in \stackrel{\circ}{H^{1}}(\Omega), \quad-\left\langle\Delta_{\varepsilon}^{\mathrm{Dir}} \Phi, \Psi\right\rangle=\int_{\Omega} \varepsilon \operatorname{grad} \Phi \operatorname{grad} \Psi
$$

- $-\Delta_{\mu}^{\mathrm{Neu}}$ is defined from $H^{1}(\Omega)$ into its dual by

$$
\forall \Phi, \Psi \in H^{1}(\Omega), \quad-\left\langle\Delta_{\mu}^{\mathrm{Neu}} \Phi, \Psi\right\rangle=\int_{\Omega} \mu \operatorname{grad} \Phi \operatorname{grad} \Psi .
$$

We end this section by a regularity result for the divergence, see also [8].

Theorem 1.1. If $\boldsymbol{u}$ solves (1.5) with $\boldsymbol{f}$ in $L^{2}(\Omega)^{3}$, then $\operatorname{div} \varepsilon \boldsymbol{u}$ belongs to $\stackrel{\circ}{H}^{1}(\Omega)$. If $\boldsymbol{u}$ solves (1.6) with $\boldsymbol{h}$ in $L^{2}(\Omega)^{3}$, then $\operatorname{div} \mu \boldsymbol{u}$ belongs to $H^{1}(\Omega)$.

Proof. Let $\boldsymbol{u}$ be solution of (1.5). Taking as test functions $\boldsymbol{v}=\operatorname{grad} \bar{\Phi}$ with $\Phi$ in the domain $D\left(\Delta_{\varepsilon}^{\text {Dir }}\right)$ of $\Delta_{\varepsilon}^{\text {Dir }}$ we obtain

$$
\forall \Phi \in D\left(\Delta_{\varepsilon}^{\text {Dir }}\right), \quad\left\langle\operatorname{div} \varepsilon \boldsymbol{u}, \Delta_{\varepsilon}^{\text {Dir }} \Phi+\omega^{2} \Phi\right\rangle_{\Omega}=\langle\boldsymbol{f}, \operatorname{grad} \Phi\rangle_{\Omega} .
$$

Let $q$ be a solution of the Dirichlet problem (if $\omega^{2}$ is an eigenvalue of $-\Delta_{\varepsilon}^{\text {Dir }}$ the above equation ensures the solvability of this problem)

$$
\forall \Psi \in \stackrel{\circ}{H^{1}}(\Omega), \quad-\langle\varepsilon \operatorname{grad} q, \operatorname{grad} \Psi\rangle_{\Omega}+\left\langle\omega^{2} q, \Psi\right\rangle_{\Omega}=\langle\boldsymbol{f}, \operatorname{grad} \Psi\rangle_{\Omega} .
$$

Whence

$$
\forall \Phi \in D\left(\Delta_{\varepsilon}^{\mathrm{Dir}}\right), \quad\left\langle q, \Delta_{\varepsilon}^{\mathrm{Dir}} \Phi+\omega^{2} \Phi\right\rangle_{\Omega}=\langle\boldsymbol{f}, \operatorname{grad} \Phi\rangle_{\Omega}
$$


Thus div $\varepsilon \boldsymbol{u}-q$ is orthogonal to the range of $\Delta_{\varepsilon}^{\text {Dir }}+\omega^{2}$, therefore is either 0 or an eigenvector of $-\Delta_{\varepsilon}^{\text {Dir }}$ associated with $\omega^{2}$. Either way, $\operatorname{div} \varepsilon \boldsymbol{u}-q$ belongs to $H^{1}(\Omega)$, hence $\operatorname{div} \varepsilon \boldsymbol{u}$ too. The proof for the "magnetic" problem (1.6) is similar.

\section{The Closure of Piecewise-Smooth functions in $\boldsymbol{X}_{N}(\Omega, \varepsilon)$ And $\boldsymbol{X}_{T}(\Omega, \mu)$}

It is clear that the bilinear forms associated with problems (1.5) and (1.6) are coercive on $\boldsymbol{X}_{N}(\Omega, \varepsilon)$ and $\boldsymbol{X}_{T}(\Omega, \mu)$ respectively. When $\varepsilon$ is smooth, it is proved in [7] that $\boldsymbol{X}_{N}(\Omega, \varepsilon) \cap \boldsymbol{H}^{1}(\Omega)$ is a closed subspace of $\boldsymbol{X}_{N}(\Omega, \varepsilon)$. In our situation, the corresponding spaces are

$$
\boldsymbol{H}_{N}(\Omega, \varepsilon):=\boldsymbol{X}_{N}(\Omega, \varepsilon) \cap P \boldsymbol{H}^{1}(\Omega, \mathscr{P}) \quad \text { and } \quad \boldsymbol{H}_{T}(\Omega, \mu):=\boldsymbol{X}_{T}(\Omega, \mu) \cap P \boldsymbol{H}^{1}(\Omega, \mathscr{P}) .
$$

From (1.4) and (1.3), we immediately obtain

$$
\begin{array}{rlrl}
\boldsymbol{H}_{N}(\Omega, \varepsilon)=\left\{\boldsymbol{u} \in L^{2}(\Omega)^{3} \mid\right. & \boldsymbol{u}_{j} \in \boldsymbol{H}^{1}\left(\Omega_{j}\right), & \\
& {[\boldsymbol{u} \times \boldsymbol{n}]_{F}=0, \quad[\varepsilon \boldsymbol{u} \cdot \boldsymbol{n}]_{F}=0,} & & \forall F \in \mathscr{F}_{\text {int }} \\
& \boldsymbol{u} \times\left.\boldsymbol{n}\right|_{F}=0, & & \left.\forall F \in \mathscr{F}_{\text {ext }}\right\}
\end{array}
$$

and

$$
\begin{aligned}
& \boldsymbol{H}_{T}(\Omega, \mu)=\left\{\boldsymbol{u} \in L^{2}(\Omega)^{3} \mid \quad \boldsymbol{u}_{j} \in \boldsymbol{H}^{1}\left(\Omega_{j}\right),\right. \\
& {[\boldsymbol{u} \times \boldsymbol{n}]_{F}=0, \quad[\mu \boldsymbol{u} \cdot \boldsymbol{n}]_{F}=0, \quad \forall F \in \mathscr{F}_{\mathrm{int}}} \\
& \left.\left.\boldsymbol{u} \cdot \boldsymbol{n}\right|_{F}=0, \quad \forall F \in \mathscr{F}_{\text {ext }}\right\} \text {. }
\end{aligned}
$$

In this section we are going to prove that not only $\boldsymbol{H}_{N}(\Omega, \varepsilon)$ is closed in $\boldsymbol{X}_{N}(\Omega, \varepsilon)$, but still $\boldsymbol{H}_{N}(\Omega, \varepsilon)$ is the closure in $\boldsymbol{X}_{N}(\Omega, \varepsilon)$ of piecewise-smooth functions. To this aim, let us introduce for any $s, 1 \leq s \leq \infty$, the spaces $\boldsymbol{H}_{N}^{s}(\Omega, \varepsilon)$ and $\boldsymbol{H}_{T}^{s}(\Omega, \mu)$ :

$$
\boldsymbol{H}_{N}^{s}(\Omega, \varepsilon):=\boldsymbol{X}_{N}(\Omega, \varepsilon) \cap P \boldsymbol{H}^{s}(\Omega, \mathscr{P}) \quad \text { and } \quad \boldsymbol{H}_{T}^{s}(\Omega, \mu):=\boldsymbol{X}_{T}(\Omega, \mu) \cap P \boldsymbol{H}^{s}(\Omega, \mathscr{P}) .
$$

Of course their elements are the piecewise- $\boldsymbol{H}^{s}$ fields satisfying the boundary and transmission conditions of (2.1) and (2.2).

Our main result in this section is:

Theorem 2.1. The closure of $\boldsymbol{H}_{N}^{\infty}(\Omega, \varepsilon)$ in $\boldsymbol{X}_{N}(\Omega, \varepsilon)$ is $\boldsymbol{H}_{N}(\Omega, \varepsilon)$, and the closure of $\boldsymbol{H}_{T}^{\infty}(\Omega, \mu)$ in $\boldsymbol{X}_{T}(\Omega, \mu)$ is $\boldsymbol{H}_{T}(\Omega, \mu)$.

The proof follows from a succession of lemmas.

Lemma 2.2. Let $C_{N}=\max _{j}\left\{\varepsilon_{j}^{-1}, \varepsilon_{j} \mu_{j}\right\}$ and $C_{T}=\max _{j}\left\{\mu_{j}^{-1}, \varepsilon_{j} \mu_{j}\right\}$. Then for any $\boldsymbol{v} \in \boldsymbol{H}_{N}^{2}(\Omega, \varepsilon)$ there holds

$$
\int_{\Omega} \varepsilon|\operatorname{grad} \boldsymbol{v}|^{2} \leq C_{N} \int_{\Omega}\left(\mu^{-1}|\operatorname{curl} \boldsymbol{v}|^{2}+|\operatorname{div} \varepsilon \boldsymbol{v}|^{2}\right),
$$

and for any $\boldsymbol{v} \in \boldsymbol{H}_{T}^{2}(\Omega, \mu)$ there holds

$$
\int_{\Omega} \mu|\operatorname{grad} \boldsymbol{v}|^{2} \leq C_{T} \int_{\Omega}\left(\varepsilon^{-1}|\operatorname{curl} \boldsymbol{v}|^{2}+|\operatorname{div} \mu \boldsymbol{v}|^{2}\right) .
$$


Note that the left hand sides of (2.3) and (2.4) are the bilinear forms of the operators $\Delta_{\varepsilon}^{\text {Dir }}$ and $\Delta_{\mu}^{\text {Neu }}$ respectively and that their right hand sides are the Maxwell bilinear forms, $c f .(1.5)$ and (1.6).

Proof. For any $j$ and any $\boldsymbol{v} \in H^{2}\left(\Omega_{j}\right)$ two successive integrations by parts yield:

$$
\begin{aligned}
\int_{\Omega_{j}} \varepsilon_{j}|\operatorname{grad} \boldsymbol{v}|^{2}= & -\int_{\Omega_{j}} \varepsilon_{j} \Delta \boldsymbol{v} \cdot \boldsymbol{v}+\int_{\partial \Omega_{j}} \varepsilon_{j} \partial_{n} \boldsymbol{v} \cdot \boldsymbol{v} \\
= & \int_{\Omega_{j}} \varepsilon_{j}\left(|\operatorname{curl} \boldsymbol{v}|^{2}+|\operatorname{div} \boldsymbol{v}|^{2}\right) \\
& +\int_{\partial \Omega_{j}} \varepsilon_{j}\left(\partial_{n} \boldsymbol{v} \cdot \boldsymbol{v}-(\operatorname{curl} \boldsymbol{v} \times \boldsymbol{n}) \cdot \boldsymbol{v}-\operatorname{div} \boldsymbol{v}(\boldsymbol{v} \cdot \boldsymbol{n})\right) .
\end{aligned}
$$

On each face of $\partial \Omega_{j}$, let us denote by $v_{n}$ the normal component $\boldsymbol{v} \cdot \boldsymbol{n}$ of $\boldsymbol{v}$ and by $\boldsymbol{v} \boldsymbol{v}_{\top}$ its tangential component $\boldsymbol{v}-v_{n} \boldsymbol{n}$. The tangential parts of the gradient and of the divergence are denoted by $\operatorname{grad}_{\mathrm{\top}}$ and $\operatorname{div}_{\top}$. Using that the faces of $\Omega_{j}$ are plane and relying in particular on the identity $-\operatorname{curl} \boldsymbol{v} \times \boldsymbol{n}=\operatorname{grad}_{\top} v_{n}-\partial_{n} \boldsymbol{v}_{\top}$ which holds on each face, we arrive at

$$
\int_{\Omega_{j}} \varepsilon_{j}|\operatorname{grad} \boldsymbol{v}|^{2}=\int_{\Omega_{j}} \varepsilon_{j}\left(|\operatorname{curl} \boldsymbol{v}|^{2}+|\operatorname{div} \boldsymbol{v}|^{2}\right)+\int_{\partial \Omega_{j}} \operatorname{grad}_{\top}\left(\varepsilon_{j} v_{n}\right) \cdot \boldsymbol{v}_{\top}-\operatorname{div} \top \boldsymbol{v}_{\top}\left(\varepsilon_{j} v_{n}\right) .
$$

If $\boldsymbol{v}$ belongs to $P \boldsymbol{H}^{2}(\Omega, \mathscr{P})$ and is such that for any interface $F \in \mathscr{F}$ int,$[\boldsymbol{v} \times \boldsymbol{n}]=0$, we deduce from the above equality that

$$
\begin{aligned}
\int_{\Omega} \varepsilon|\operatorname{grad} \boldsymbol{v}|^{2}= & \int_{\Omega} \varepsilon\left(|\operatorname{curl} \boldsymbol{v}|^{2}+|\operatorname{div} \boldsymbol{v}|^{2}\right)+\sum_{F \in \mathscr{F}_{\text {ext }}} \int_{F} \operatorname{grad}_{\top}\left(\varepsilon v_{n}\right) \cdot \boldsymbol{v}_{\top}-\operatorname{div} \top \boldsymbol{v}_{\top}\left(\varepsilon v_{n}\right) \\
& +\sum_{F \in \mathscr{F}_{\text {int }}} \int_{F} \operatorname{grad}_{\top}\left[\varepsilon v_{n}\right]_{F} \cdot \boldsymbol{v}_{\top}-\operatorname{div}_{\top} \boldsymbol{v}_{\top}\left[\varepsilon v_{n}\right]_{F} .
\end{aligned}
$$

Thus, if $\boldsymbol{v} \in \boldsymbol{H}_{N}^{2}(\Omega, \varepsilon), \int_{\Omega} \varepsilon|\operatorname{grad} \boldsymbol{v}|^{2}$ is equal to $\int_{\Omega} \varepsilon\left(|\operatorname{curl} \boldsymbol{v}|^{2}+|\operatorname{div} \boldsymbol{v}|^{2}\right)$ and similarly, if $\boldsymbol{v} \in \boldsymbol{H}_{T}^{2}(\Omega, \mu)$, $\int_{\Omega} \mu|\operatorname{grad} \boldsymbol{v}|^{2}$ is equal to $\int_{\Omega} \mu\left(|\operatorname{curl} \boldsymbol{v}|^{2}+|\operatorname{div} \boldsymbol{v}|^{2}\right)$. Estimates (2.3) and (2.4) are now straightforward.

Now we are going to prove density results. For this, we go through several steps.

Lemma 2.3. Let $\omega$ be a bounded sector of radius 1 in $\mathbb{R}^{2}$ and let $r$ be the distance to its vertex. Let $h$ belong to $H^{1}(\omega)$. Then $r^{\alpha} h$ tends to $h$ in $H^{1}(\omega)$ as $\alpha \rightarrow 0$.

Proof. By the dominated convergence theorem, we obtain immediately that $r^{\alpha} h, r^{\alpha} \partial_{x} h$ and $r^{\alpha} \partial_{y} h$ tend to $h$, $\partial_{x} h$ and $\partial_{y} h$ respectively in $L^{2}(\omega)$ as $\alpha \rightarrow 0$. It remains to prove that $h \partial_{r} r^{\alpha}$ tends to 0 in $L^{2}(\omega)$ as $\alpha \rightarrow 0$.

The difficulty lying in $r=0$, we can assume that $h=0$ on $r=1$. With the help of an integration by parts, we obtain

$$
\begin{aligned}
\int_{0}^{1}\left|h \partial_{r} r^{\alpha}\right|^{2} r \mathrm{~d} r=\frac{1}{2} \int_{0}^{1} \alpha h^{2} \partial_{r} r^{2 \alpha} \mathrm{d} r & =-\int_{0}^{1} \alpha h \partial_{r} h r^{2 \alpha} \mathrm{d} r \\
& =-\int_{0}^{1} \alpha r^{\alpha-1} h r^{\alpha} \partial_{r} h r \mathrm{~d} r
\end{aligned}
$$

from which we deduce

Thus, setting

$$
\left\|h \partial_{r} r^{\alpha}\right\|_{L^{2}(\omega)}^{2} \leq\left\|h \partial_{r} r^{\alpha}\right\|_{L^{2}(\omega)}\left\|r^{\alpha} \partial_{r} h\right\|_{L^{2}(\omega)} .
$$

$$
X(\alpha)=\left\|h \partial_{r} r^{\alpha}\right\|_{L^{2}(\omega)}^{2}
$$


we have obtained that $X(\alpha)$ is bounded as $\alpha \rightarrow 0$. Similarly as above, we have

$$
X(\alpha)-2 X\left(\frac{\alpha}{2}\right)=-\int_{\omega} \alpha r^{\alpha-1} h\left(r^{\alpha} \partial_{r} h-\partial_{r} h\right) \mathrm{d} x \mathrm{~d} y,
$$

from which we deduce

$$
\left|X(\alpha)-2 X\left(\frac{\alpha}{2}\right)\right| \leq \sqrt{X(\alpha)}\left\|r^{\alpha} \partial_{r} h-\partial_{r} h\right\|_{L^{2}(\omega)} .
$$

Thus, $|X(\alpha)-2 X(\alpha / 2)|$ tends to 0 as $\alpha \rightarrow 0$. As $X(\alpha)$ is bounded, we can deduce from this what we wanted, i.e. that $X(\alpha) \rightarrow 0$.

Lemma 2.4. Let $\omega$ be as in Lemma (2.3) and let $\chi=\chi(r)$ a smooth function in $\mathscr{C}_{0}^{\infty}(-1,1)$ equal to 1 in a neighborhood of 0 . Let $h$ belong to $H^{1}(\omega)$. Then $h$ belongs to the closure in $H^{1}(\omega)$ of the set

$$
S(h):=\left\{r^{\alpha}(1-\chi(n r)) h \mid \alpha \in(0,1), n \in \mathbb{N}\right\} .
$$

Proof. With Lemma (2.3) we have only to prove that we can choose $\alpha$ and $n$ so that the norm of $r^{\alpha} \chi(n r) h$ in $H^{1}(\omega)$ is as small as we want. Obviously, $r^{\alpha} \chi(n r) h, r^{\alpha} \chi(n r) \partial_{x} h$ and $r^{\alpha} \chi(n r) \partial_{y} h$ tend to 0 in $L^{2}(\omega)$ as $n \rightarrow \infty$ uniformly in $\alpha \in(0,1)$. From the proof of Lemma 2.3, we have that $h \partial_{r} r^{\alpha}$ tends to 0 , thus $\chi(n r) h \partial_{r} r^{\alpha}$ tends to 0 in $L^{2}(\omega)$ as $\alpha \rightarrow 0$, uniformly in $n$. It remains to evaluate the norm of $r^{\alpha} h \partial_{r} \chi(n r)$ in $L^{2}(\omega)$. We start from the estimate

$$
\exists C>0, \quad \forall r \in(0,1), \forall n \in \mathbb{N}, \quad\left|\partial_{r} \chi(n r)\right| \leq \frac{C}{r} .
$$

Then, as the support of $\partial_{r} \chi(n r)$ is contained in $\left(0, \frac{1}{n}\right)$, we have

$$
\left\|r^{\alpha} h \partial_{r} \chi(n r)\right\|_{L^{2}(\omega)} \leq n^{-\alpha / 2}\left\|r^{\alpha / 2} h \partial_{r} \chi(n r)\right\|_{L^{2}(\omega)} \leq C n^{-\alpha / 2}\left\|r^{-1+\alpha / 2} h\right\|_{L^{2}(\omega)} .
$$

Since, for any $\alpha>0$, by Hardy's inequality, $r^{-1+\alpha / 2} h$ belongs to $L^{2}(\omega)$, for any fixed $\alpha$, we can choose $n$ so that $\left\|r^{\alpha} h \partial_{r} \chi(n r)\right\|_{L^{2}(\omega)}$ is as small as we want.

As a straightforward corollary of the previous lemma, we obtain the corresponding result in $\mathbb{R}^{3}$ :

Lemma 2.5. Let $W=\omega \times I$ where $\omega$ is a plane sector and $I$ an open interval. Let $h$ belong to $H^{1}(W)$. Then $h$ belongs to the closure in $H^{1}(W)$ of the set $S(h)$ defined by (2.5) where $r$ is still the distance to the vertex in $\omega$.

Lemma 2.6. Let $\Omega_{j}$ be a polyhedral partition of $\Omega$ and let $\Sigma$ be the skeleton formed by the union of the closed edges of all the $\Omega_{j}$. Then the subspace of $\boldsymbol{H}_{N}^{\infty}(\Omega, \varepsilon)$ of the fields which are zero on $\Sigma$, is dense in $\boldsymbol{H}_{N}(\Omega, \varepsilon)$, and similarly for the spaces $\boldsymbol{H}_{T}(\Omega, \mu)$.

Proof. Let $\boldsymbol{h} \in \boldsymbol{H}_{N}(\Omega, \varepsilon)$ and $\varepsilon>0$. The proof of the existence of a $\tilde{\boldsymbol{h}} \in \boldsymbol{H}_{N}^{\infty}(\Omega, \varepsilon)$ such that $\tilde{\boldsymbol{h}}=0$ on $\Sigma$ and $\|\boldsymbol{h}-\tilde{\boldsymbol{h}}\|_{P \boldsymbol{H}^{1}(\Omega, \mathscr{P})}<\varepsilon$ is organized in three steps.

Step 1. Let $\chi$ be a function like in Lemmas 2.4 and 2.5. For each vertex $S \in \Sigma$ let $\rho_{S}$ be the distance to $S$. Then $\chi\left(n \rho_{S}\right) \boldsymbol{h}$ tends to 0 in $P \boldsymbol{H}^{1}(\Omega, \mathscr{P})$ for each vertex $S$ as $n \rightarrow \infty$. Thus we can choose $n$ large enough so that

$$
\boldsymbol{h}_{1}:=\boldsymbol{h}-\sum_{S} \chi\left(n \rho_{S}\right) \boldsymbol{h} \quad \text { is such that }\left\|\boldsymbol{h}-\boldsymbol{h}_{1}\right\|_{P \boldsymbol{H}^{1}(\Omega, \mathscr{P})}<\varepsilon / 4 .
$$

Then we can apply Lemma 2.5 to $\boldsymbol{h}_{1}$ in the neighborhood of each edge in $\Sigma$, and we obtain a new field $\boldsymbol{h}_{2}$ in $\boldsymbol{H}_{N}^{1}(\Omega, \varepsilon)$ such that

$$
\boldsymbol{h}_{2}=0 \text { in a neighborhood } \mathscr{V} \text { of } \Sigma \text { and }\left\|\boldsymbol{h}-\boldsymbol{h}_{2}\right\|_{P \boldsymbol{H}^{1}(\Omega, \mathscr{P})}<\varepsilon / 2 .
$$


Step 2. Let $\mathscr{V}_{0}$ be a neighborhood of $\Sigma$ such that $\overline{\mathscr{V}}_{0} \subset \mathscr{V}$. We can then introduce independent lifting of traces $R_{F}$ on each face $F \in \mathscr{F}_{\text {int }} \cup \mathscr{F}_{\text {ext }}$ acting from the subspace of $H^{1 / 2}(F)$ of functions $g$ which are zero on $\mathscr{V}_{0}$, into $P \boldsymbol{H}^{1}(\Omega, \mathscr{P})$, so that $R_{F}(g)$ is zero in a neighborhood of all the other faces. With these liftings, we can construct a lifting $R_{N}$ of the trace and jump operator $\gamma_{N}$

$$
\begin{array}{rlc}
\gamma_{N}: P \boldsymbol{H}^{1}(\Omega, \mathscr{P}) & \longrightarrow \prod_{F \in \mathscr{F}_{\text {ext }}} H^{1 / 2}(F)^{2} \times \quad \prod_{F \in \mathscr{F}_{\mathrm{int}}} H^{1 / 2}(F)^{3} \\
\boldsymbol{v} & \longmapsto\left(\boldsymbol{g}_{\top, F}=\boldsymbol{v} \times\left.\boldsymbol{n}\right|_{F}, \quad \boldsymbol{g}_{\top, F}=[\boldsymbol{v} \times \boldsymbol{n}]_{F}, \quad \boldsymbol{g}_{n, F}=[\varepsilon \boldsymbol{v} \cdot \boldsymbol{n}]_{F}\right),
\end{array}
$$

such that $\gamma_{N} R_{N} \boldsymbol{g}=\boldsymbol{g}$ for all set of traces and jumps which are zero on $\mathscr{V}_{0}$. Let $C_{R}$ be the norm of $R_{N}$.

Step 3. We regularize $\boldsymbol{h}_{2}$ in each $\Omega_{j}$ by convolution by a regularizing sequence $\chi_{n}$. For $n$ large enough, the regularized field $\boldsymbol{h}_{3}$ is zero on $\mathscr{V}_{0}$ and

$$
\left\|\boldsymbol{h}_{2}-\boldsymbol{h}_{3}\right\|_{P \boldsymbol{H}^{1}(\Omega, \mathscr{P})}<\varepsilon / 4 \text { and }\left\|\gamma_{N} \boldsymbol{h}_{3}\right\|_{P \boldsymbol{H}^{1 / 2}}<\varepsilon /\left(4 C_{R}\right) .
$$

Setting $\tilde{\boldsymbol{h}}=\boldsymbol{h}_{3}-R_{N} \gamma_{N} \boldsymbol{h}_{3}$ yields the desired approximation of $\boldsymbol{h}$. The proof for the other boundary conditions is similar.

Now, Theorem 2.1 is clearly a consequence of Lemmas 2.2 and 2.6 .

\section{Singularities of the VARiational SPACES}

In this section we establish continuous decompositions of the spaces $\boldsymbol{X}_{N}(\Omega, \varepsilon)$ and $\boldsymbol{X}_{T}(\Omega, \mu)$ into a $\boldsymbol{H}^{1}$ or $P \boldsymbol{H}^{1}$ field and a gradient. Such a decomposition is well known for the homogeneous Maxwell's equations, i.e. when $\varepsilon$ and $\mu$ are constant or sufficiently regular (e.g. Lipschitz) $[2-4,12,17]$, and was extended to the heterogeneous case by [5] under the assumption of two materials with a plane interface. We prove here two sorts of decompositions in our general framework.

We begin with two lemmas giving the existence of regular vector potentials:

Lemma 3.1. Let us assume that $\Omega$ is simply connected. Let $\boldsymbol{u}$ be a divergence-free $L^{2}$ field. Then there exists $\psi \in \boldsymbol{H}_{T}(\Omega, 1)$ such that $\operatorname{curl} \psi=\boldsymbol{u}$.

This Lemma is simply obtained by the combination of Theorem 3.12 in [1] which yields a potential $\psi_{0}$ in $\boldsymbol{X}_{T}(\Omega, 1)$ and a decomposition of this $\psi_{0}$ in a regular $\psi \in \boldsymbol{H}_{T}(\Omega, 1)$ and a gradient according to [3]. Of course this gradient part does not contribute to the curl!

Similarly, relying on Theorem 3.17 in [1], and [3], we obtain

Lemma 3.2. Let us assume that $\Omega$ is simply connected. Let $\boldsymbol{u}$ be a divergence-free $L^{2}$ field such that $\boldsymbol{u} \cdot \boldsymbol{n}$ is zero on $\partial \Omega$. Then there exists $\psi \in \boldsymbol{H}_{N}(\Omega, 1)$ such that $\operatorname{curl} \psi=\boldsymbol{u}$.

We also introduce the following notation:

Notation 3.3. For $g=\left(g_{F}\right)_{F} \in P H^{1 / 2}\left(\mathscr{F}_{\text {int }}\right)$ and $f \in L^{2}(\Omega)$ we write

$$
-\Delta_{\varepsilon}^{\mathrm{Dir}} \Phi=f+\sum_{F \in \mathscr{F}_{\mathrm{int}}} g_{F} \otimes \delta_{F}
$$

if we have the variational formulation (1.7):

$$
\Phi \in \stackrel{\circ}{H}{ }^{1}(\Omega), \forall \Psi \in \stackrel{\circ}{H^{1}}(\Omega), \quad \int_{\Omega} \varepsilon \operatorname{grad} \Phi \operatorname{grad} \Psi=\int_{\Omega} f \Psi \mathrm{d} x+\sum_{F \in \mathscr{F}_{\text {int }}} \int_{F} g_{F} \Psi \mathrm{d} \sigma .
$$


We use the analogous notation for $\Delta_{\mu}^{\mathrm{Neu}}$ based on the variational formulation (1.8) with the same right hand side as above:

$$
\Phi \in H^{1}(\Omega), \forall \Psi \in H^{1}(\Omega), \quad \int_{\Omega} \mu \operatorname{grad} \Phi \operatorname{grad} \Psi=\int_{\Omega} f \Psi \mathrm{d} x+\sum_{F \in \mathscr{F}_{\text {int }}} \int_{F} g_{F} \Psi \mathrm{d} \sigma .
$$

Our first decomposition result yields a "regular" part in $\boldsymbol{H}^{1}(\Omega)$ and a "singular" part in the form of a gradient, which contains in particular all the jumps through the interfaces.

Theorem 3.4. Any field $\boldsymbol{v} \in \boldsymbol{X}_{T}(\Omega, \mu)$ admits a decomposition

$$
\boldsymbol{v}=\psi+\operatorname{grad} \Phi,
$$

where $\psi \in \boldsymbol{H}_{T}(\Omega, 1)$ and $\Phi \in H^{1}(\Omega)$ satisfies $-\Delta_{\mu}^{\mathrm{Neu}} \Phi=f+\sum_{\mathscr{F}_{\text {int }}} g_{F} \otimes \delta_{F}$ with $f \in L^{2}(\Omega), g \in P H^{1 / 2}\left(\mathscr{F}_{\text {int }}\right)$. Similarly any $\boldsymbol{v} \in \boldsymbol{X}_{N}(\Omega, \varepsilon)$ admits a decomposition (3.1) where $\psi \in \boldsymbol{H}_{N}(\Omega, 1)$ and $\Phi \in \stackrel{\circ}{H}^{1}(\Omega)$ satisfies $-\Delta_{\varepsilon}^{\operatorname{Dir}} \Phi=f+\sum_{\mathscr{F}_{\text {int }}} g_{F} \otimes \delta_{F}$. In both cases there holds

$$
\|\psi\|_{H^{1}(\Omega)}+\|f\|_{L^{2}(\Omega)}+\|g\|_{P H^{1 / 2}\left(\mathscr{F}_{\mathrm{int}}\right)} \leq C\|\boldsymbol{v}\|_{\boldsymbol{X}} .
$$

Proof. We first note that with the help of a partition of unity, we can reduce to the case when $\Omega$ is simply connected.

Let $\boldsymbol{v} \in \boldsymbol{X}_{T}(\Omega, \mu)$. Since its curl is a $L^{2}$ divergence-free field we can apply Lemma (3.1) to $\boldsymbol{u}=\operatorname{curl} \boldsymbol{v}$ and find $\psi \in \boldsymbol{H}_{T}(\Omega, 1)$ such that $\operatorname{curl} \psi=\operatorname{curl} \boldsymbol{v}$. Then $\boldsymbol{v}-\psi$ is a curl-free field. As $\Omega$ is simply connected, this is a gradient: there exists $\Phi \in H^{1}(\Omega)$ such that $\boldsymbol{v}-\psi=\operatorname{grad} \Phi$. Obviously $\Phi$ satisfies

$$
\forall \Psi \in H^{1}(\Omega), \quad \int_{\Omega} \mu \operatorname{grad} \Phi \operatorname{grad} \Psi=\int_{\Omega} \mu(\boldsymbol{v}-\psi) \operatorname{grad} \Psi \mathrm{d} x,
$$

which enters the framework of Notation (3.3) with $f=-\operatorname{div} \mu \boldsymbol{v}+\widetilde{\operatorname{div}} \mu \psi$, where the operator $\widetilde{\operatorname{div}}$ is the divergence in $\cup_{j} \Omega_{j}$ (and not in $\Omega$ ), and for all $F \in \mathscr{F}_{\text {int }}, g_{F}=-[\mu]_{F} \psi \cdot \boldsymbol{n}$.

Now, if $\boldsymbol{v} \in \boldsymbol{X}_{N}(\Omega, \varepsilon)$, we note that $\operatorname{curl} \boldsymbol{v}$ satisfies also $\operatorname{curl} \boldsymbol{v} \cdot \boldsymbol{n}=0$ on $\partial \Omega$. Thus we can apply Lemma (3.2) to obtain $\psi \in \boldsymbol{H}_{N}(\Omega, 1)$ such that $\operatorname{curl} \psi=\operatorname{curl} \boldsymbol{v}$. Then, as above, there exists $\Phi \in H^{1}(\Omega)$ such that $\boldsymbol{v}-\psi=$ $\operatorname{grad} \Phi$. Since $(\boldsymbol{v}-\psi) \times \boldsymbol{n}=0$ on $\partial \Omega, \Phi$ belongs to $\stackrel{\circ}{H}^{1}(\Omega)$ and the proof ends as above.

Our second decomposition result is more in the spirit of the splittings given in [3,4,12] and [5]. It consists in obtaining a "regular" part in $\boldsymbol{H}_{T}(\Omega, \mu)$ or $\boldsymbol{H}_{N}(\Omega, \varepsilon)$ instead of $\boldsymbol{H}_{T}(\Omega, 1)$ or $\boldsymbol{H}_{N}(\Omega, 1)$. For the assumptions and the proof of this statement we use some facts and terminology about the behavior of the operators $\Delta_{\mu}^{\text {Neu }}$ and $\Delta_{\varepsilon}^{\text {Dir }}$ with respect to the corners and edges of $\Omega$ and of its subdomains $\Omega_{j}$ which we describe in the next section.

\section{Theorem 3.5.}

(i) Let us assume that the operator $\Delta_{\mu}^{\mathrm{Neu}}$ has no edge exponent equal to 1 and no corner exponent equal to $1 / 2$. Then any field $\boldsymbol{v} \in \boldsymbol{X}_{T}(\Omega, \mu)$ admits a decomposition

$$
\boldsymbol{v}=\boldsymbol{w}+\operatorname{grad} \Phi_{0},
$$

where $\boldsymbol{w} \in \boldsymbol{H}_{T}(\Omega, \mu)$ and $\Phi_{0} \in H^{1}(\Omega)$ satisfies $-\Delta_{\mu}^{\mathrm{Neu}} \Phi_{0} \in L^{2}(\Omega)$.

(ii) Let us assume that the operator $\Delta_{\varepsilon}^{\text {Dir }}$ has no edge exponent equal to 1 and no corner exponent equal to 1/2. Then any field $\boldsymbol{v} \in \boldsymbol{X}_{N}(\Omega, \varepsilon)$ admits a decomposition (3.3) where $\boldsymbol{w} \in \boldsymbol{H}_{N}(\Omega, \varepsilon)$ and $\Phi_{0} \in \stackrel{\circ}{H}^{1}(\Omega)$ satisfies $-\Delta_{\varepsilon}^{\mathrm{Dir}} \Phi_{0} \in L^{2}(\Omega)$. 
Proof. (i) We start from the first decomposition (3.1) and split $\Phi$ into two parts, each belonging to $H^{1}(\Omega)$ (see Th. 4.1):

$$
\Phi=\Phi_{0}+\Phi_{1}, \quad \text { with } \quad \Delta_{\mu}^{\mathrm{Neu}} \Phi_{0} \in L^{2}(\Omega) \quad \text { and } \quad \Phi_{1} \in P H^{2}(\Omega, \mathscr{P}) .
$$

We then set $\boldsymbol{w}=\psi+\operatorname{grad} \Phi_{1}$ which belongs to $P \boldsymbol{H}^{1}(\Omega, \mathscr{P})$. Since $\Delta_{\mu}^{\mathrm{Neu}} \Phi_{0} \in L^{2}(\Omega)$, grad $\Phi_{0}$ belongs to $\boldsymbol{X}_{T}(\Omega, \mu)$. Thus $\boldsymbol{w}$ also belongs to $\boldsymbol{X}_{T}(\Omega, \mu)$, therefore to $\boldsymbol{H}_{T}(\Omega, \mu)$. The proof for (ii) is similar.

\section{LAPLACE INTERFACE SINGULARITIES}

As a synthesis of the thorough treatment of bidimensional interface problems in [18] and of tridimensional monodomain boundary value problems in [9], we briefly present in this section the regularity and splitting results for the Laplace interface operators $\Delta_{\varepsilon}^{\text {Dir }}$ and $\Delta_{\mu}^{\mathrm{Neu}}$.

The notion of corner and edge is clear for a polyhedron in $\mathbb{R}^{3}$. Concerning $\Omega$ with its polyhedral partition $\mathscr{P}$, we call corner of $(\Omega, \mathscr{P})$ any point $\boldsymbol{c}$ which is a corner of (at least) one of the $\Omega_{j}$ and edge any segment $\boldsymbol{e}$ which is an edge of one of the $\Omega_{j}$ and either disjoint from the other $\Omega_{k}$ or contained in one of their edges.

Let us give an illustrative example: $\Omega_{1}$ and $\Omega_{2}$ are the unit cubes $(0,1)^{3}$ and $(-1,0) \times(0,1)^{2}$, and $\Omega_{3}$ is the parallelepiped $(-1,1)^{2} \times(-1,0)$. Finally $\Omega$ is the interior of $\bar{\Omega}_{1} \cup \bar{\Omega}_{2} \cup \bar{\Omega}_{3}$. The corners are the corners of $\Omega$ and the points $\boldsymbol{c}_{1}=(0,0,0), \boldsymbol{c}_{2}=(0,1,0), \boldsymbol{c}_{3}=(0,1,1), \boldsymbol{c}_{4}=(0,0,1), \boldsymbol{c}_{5}=(1,1,0)$ and $\boldsymbol{c}_{6}=(-1,1,0)$. With the two other corners $\boldsymbol{c}_{7}=(1,0,0)$ and $\boldsymbol{c}_{8}=(-1,0,0)$, the interface edges are $\left[\boldsymbol{c}_{1}, \boldsymbol{c}_{2}\right]$ (triple), $\left[\boldsymbol{c}_{2}, \boldsymbol{c}_{3}\right],\left[\boldsymbol{c}_{3}, \boldsymbol{c}_{4}\right]$, $\left[\boldsymbol{c}_{4}, \boldsymbol{c}_{1}\right],\left[\boldsymbol{c}_{1}, \boldsymbol{c}_{7}\right],\left[\boldsymbol{c}_{7}, \boldsymbol{c}_{5}\right],\left[\boldsymbol{c}_{5}, \boldsymbol{c}_{2}\right],\left[\boldsymbol{c}_{1}, \boldsymbol{c}_{8}\right],\left[\boldsymbol{c}_{8}, \boldsymbol{c}_{6}\right],\left[\boldsymbol{c}_{6}, \boldsymbol{c}_{2}\right]$ (double).

Note that it is possible to have corners and edges contained in the interior of $\Omega$. This would happen if we add to the example above the fourth domain $\Omega_{4}=(-1,1) \times(-1,0) \times(0,1)$. Then $\Omega$ is the cube $(-1,1)^{3}, \boldsymbol{c}_{1}$ is an interior corner and is the end of interior edges.

The general principle governing the properties of the operators $\Delta_{\varepsilon}^{\text {Dir }}$ and $\Delta_{\mu}^{\text {Neu }}$ relies on the knowledge of the exponents $\lambda$ attached to each corner and edge of $(\Omega, \mathscr{P})$, which are the (here real) numbers such that there exist non-polynomial pseudo-homogeneous solutions of degree $\lambda$ to model problems on the cones or sectors $\Gamma$ associated with the corresponding corner or edge.

\subsection{Corner exponents}

If $\boldsymbol{c}$ is one fixed corner of $(\Omega, \mathscr{P})$, we shall use polar coordinates $(\rho, \vartheta)$ centered at $\boldsymbol{c}$ and denote by $\Gamma_{\boldsymbol{c}}$ the polyhedral cone which coincides with $\Omega$ near $c$. To each $\Omega_{j}$ containing $c$ there corresponds a unique cone $\Gamma_{c, j} \subset \Gamma_{c}$ and we denote by $\mathscr{F}_{\text {int }, c}$ the set of interior (to $\Gamma_{c}$ ) faces of $\partial \Gamma_{c, j}$.

We then denote by $G_{c}$ the intersection of $\Gamma_{c}$ with the unit sphere. For any $\lambda \in \mathbb{C}$, let us set

$$
S^{\lambda}\left(\Gamma_{c}\right)=\left\{\Psi=\rho^{\lambda} \sum_{q=0}^{Q} \log ^{q} \rho \psi_{q}(\vartheta) \mid \psi_{q} \in H^{1}\left(G_{c}\right)\right\},
$$

which is the space of pseudo-homogeneous functions whose angular regularity is compatible with the $H^{1}$ regularity of variational solutions. Fitting to the operator $\Delta_{\varepsilon}^{\text {Dir }}$, we consider the subspace $S_{0}^{\lambda}\left(\Gamma_{c}\right)$ of $S^{\lambda}\left(\Gamma_{c}\right)$ of the functions which are zero on $\partial \Gamma_{c}$. When $\lambda \in \mathbb{N}$, we need two further families of polynomial spaces (which are reduced to $\{0\}$ if $\lambda \notin \mathbb{N}$ ) corresponding to solutions and right hand sides respectively. Let $P_{0}^{\lambda}\left(\Gamma_{c}, \mathscr{P}\right)$ be the subspace of $S_{0}^{\lambda}\left(\Gamma_{c}\right)$ of the functions which are polynomial in each $\Gamma_{c, j}$ and let $Q^{\lambda}\left(\Gamma_{c}, \mathscr{P}\right)$ be the space of the couples $(f, g)$ with $f$ homogeneous polynomial of degree $\lambda-2$ in each $\Gamma_{c, j}$ and $g=\left(g_{F}\right)_{F \in \mathscr{F}_{\text {int }, c}}$ with $g_{F}$ homogeneous polynomial of degree $\lambda-1$ in the interface $F$.

The set $\Lambda_{\varepsilon}^{\text {Dir }}\left(\Gamma_{c}\right)$ of the corner exponents of the Dirichlet operator $\Delta_{\varepsilon}^{\text {Dir }}$ is then the set of the $\lambda \in \mathbb{C}$ such that there exist solutions $\Psi \in S_{0}^{\lambda}\left(\Gamma_{c}\right) \backslash P_{0}^{\lambda}\left(\Gamma_{c}, \mathscr{P}\right)$ to

$$
-\Delta_{\varepsilon}^{\mathrm{Dir}} \Psi=f+\sum_{F \in \mathscr{F}_{\text {int }, c}} g_{F} \otimes \delta_{F}, \quad \text { with } \quad(f, g) \in Q^{\lambda}\left(\Gamma_{c}, \mathscr{P}\right)
$$


[cf. Notation (3.3)]. We denote the space of these solutions by $Z_{\mathrm{Dir}}^{\lambda}\left(\Gamma_{c}, \varepsilon\right)$. The sets $\Lambda_{\mu}^{\mathrm{Neu}}\left(\Gamma_{c}\right)$ and $Z_{\mathrm{Neu}}^{\lambda}\left(\Gamma_{c}, \mu\right)$ are defined similarly. Note that if $c$ is an interior corner, the spaces $S_{0}^{\lambda}\left(\Gamma_{c}\right)$ and $S^{\lambda}\left(\Gamma_{c}\right)$ coincide and there is no influence of the external boundary conditions.

Since there holds

$$
\Delta_{\varepsilon}^{\operatorname{Dir}}\left(\rho^{\lambda} \psi(\vartheta)\right)=0 \Longleftrightarrow \operatorname{div}_{\top} \varepsilon \operatorname{grad}_{\top} \psi+\lambda(\lambda+1) \varepsilon \psi=0
$$

with $\operatorname{grad}_{\mathrm{T}}$ and $\operatorname{div}_{\mathrm{T}}$ the tangential gradient and divergence on $G_{\boldsymbol{c}}$, the set of corner exponents in $\boldsymbol{c}$ is related to the spectrum of the positive Dirichlet Laplace-Beltrami operator $L_{\varepsilon, c}^{\text {Dir }}$ associated with the quadratic form

$$
(\psi, \varphi) \longmapsto\left(\operatorname{grad}_{\top} \psi, \operatorname{grad}_{\top} \varphi\right)_{\varepsilon}
$$

on the space $L^{2}\left(G_{\boldsymbol{c}}, \varepsilon\right)$ with scalar product

$$
(\psi, \varphi) \longmapsto(\psi, \varphi)_{\varepsilon}=\int_{G_{c}} \varepsilon \psi \varphi \mathrm{d} \sigma .
$$

The operator $L_{\varepsilon, c}^{\text {Dir }}$ is self-adjoint on $L^{2}\left(G_{c}, \varepsilon\right)$ with a compact inverse. Let $\nu_{1}<\nu_{2} \leq \cdots$ be its eigenvalues and $\psi_{j}$ be the corresponding eigenfunctions. Then one can show that

$$
\Lambda_{\varepsilon}^{\operatorname{Dir}}\left(\Gamma_{c}\right) \backslash \mathbb{N}=\left\{-\frac{1}{2} \pm \sqrt{\nu_{j}+\frac{1}{4}}, j \geq 1\right\} \backslash \mathbb{N}
$$

and, if $\lambda \notin \mathbb{N}$

$$
Z_{\text {Dir }}^{\lambda}\left(\Gamma_{c}, \varepsilon\right)=\operatorname{span}\left\{\rho^{\lambda} \psi_{j}(\vartheta) \mid \lambda=-\frac{1}{2} \pm \sqrt{\nu_{j}+\frac{1}{4}}\right\}
$$

The situation is similar for $\Lambda_{\mu}^{\mathrm{Neu}}\left(\Gamma_{c}\right)$ and $Z_{\mathrm{Neu}}^{\lambda}\left(\Gamma_{c}, \mu\right)$.

Relying on (4.3), we can prove that for any corner $c, 0 \notin \Lambda_{\varepsilon}^{\operatorname{Dir}}\left(\Gamma_{c}\right)$ and $0 \notin \Lambda_{\mu}^{\mathrm{Neu}}\left(\Gamma_{c}\right)$.

\subsection{Edge exponents}

Fix one edge $e$ of $\Omega$ and denote by $\Gamma_{e}$ the two-dimensional plane sector such that $\Gamma_{e} \times \mathbb{R}$ coincides with $\Omega$ in a neighbourhood of an interior point of $\boldsymbol{e}$. The polar coordinates in $\Gamma_{\boldsymbol{e}}$ are denoted $(r, \theta)$, the Cartesian coordinates in the plane of $\Gamma_{\boldsymbol{e}}$ are denoted $\boldsymbol{y}$, and $z$ is the perpendicular coordinate. To each $\Omega_{j}$ containing $\boldsymbol{e}$ there corresponds a unique sector $\Gamma_{e, j} \subset \Gamma_{e}$ and we denote by $\mathscr{F}_{\text {int, } e}$ the set of interior faces of $\partial \Gamma_{e, j}$.

Like above, we can introduce the spaces $S^{\lambda}\left(\Gamma_{e}\right), S_{0}^{\lambda}\left(\Gamma_{e}\right)$ and $P_{0}^{\lambda}\left(\Gamma_{e}, \mathscr{P}\right)$ of homogeneous functions of degree $\lambda$ in the sector $\Gamma_{e}$ and the corresponding space for the right-hand sides $Q^{\lambda}\left(\Gamma_{e}, \mathscr{P}\right)$. Then the set $\Lambda_{\varepsilon}^{\mathrm{Dir}}\left(\Gamma_{e}\right)$ of the edge exponents of the Dirichlet transmission operator is defined exactly like above as the set of the $\lambda \in \mathbb{C}$ such that there exist solutions $\Psi \in S_{0}^{\lambda}\left(\Gamma_{e}\right) \backslash P_{0}^{\lambda}\left(\Gamma_{e}, \mathscr{P}\right)$ to

$$
-\Delta_{\varepsilon}^{\mathrm{Dir}} \Psi=f+\sum_{F \in \mathscr{F}_{\mathrm{int}, e}} g_{F} \otimes \delta_{F}, \quad \text { with } \quad(f, g) \in Q^{\lambda}\left(\Gamma_{\boldsymbol{e}}, \mathscr{P}\right)
$$

where $\Delta_{\varepsilon}^{\text {Dir }}$ acting in the sector $\Gamma_{e}$ is simply the operator obtained from the corresponding three-dimensional operator by dropping the variable $z$. Thus the edge exponents are the same as the singularity exponents for two-dimensional interface problems; see $[10,15,16,19]$.

The intersection between $\Gamma_{e}$ and the unit circle being denoted $G_{e}$, with $\left(\nu_{j}\right)_{j \geq 1}$ the spectrum of the positive Laplace-Beltrami operator $L_{\varepsilon, e}^{\text {Dir }}$ associated with the quadratic form $(\psi, \varphi) \mapsto\left(\partial_{\theta} \psi, \partial_{\theta} \varphi\right)_{\varepsilon}$ on the space $L^{2}\left(G_{e}, \varepsilon\right)$, 
we have:

$$
\Lambda_{\varepsilon}^{\operatorname{Dir}}\left(\Gamma_{e}\right)=\left\{ \pm \sqrt{\nu_{j}}, j \geq 1\right\}
$$

Indeed, when $\lambda \notin \mathbb{N}$, this can be proved like (4.4) from the equivalence

$$
\Delta_{\varepsilon}^{\text {Dir }}\left(r^{\lambda} \psi(\theta)\right)=0 \quad \Longleftrightarrow \quad \partial_{\theta} \varepsilon \partial_{\theta} \psi+\lambda^{2} \varepsilon \psi=0
$$

and when $\lambda \in \mathbb{N}$, this also relies on the equality for the dimensions of the polynomial spaces

$$
\operatorname{dim} P_{0}^{\lambda}\left(\Gamma_{e}, \mathscr{P}\right)=\operatorname{dim} Q^{\lambda}\left(\Gamma_{e}, \mathscr{P}\right)=\lambda J_{e}-I_{e},
$$

where $J_{e}$ is the number of the sectors $\Gamma_{e, j}$ and $I_{e}=0$ if $e$ is an internal edge and $I_{e}=1$ if not; see Corollary 4.9 in [9].

\subsection{Regularity and singularities}

We first give a global statement, then provide a description of the singular solutions, which requires the introduction of further notations.

Theorem 4.1. Let $s>0, s \neq 1 / 2, f \in P H^{s-1}(\Omega, \mathscr{P})$ and $g \in P H^{s-1 / 2}\left(\mathscr{F}_{\text {int }}\right)$. Let $\Phi$ be the solution of the problem

$$
-\Delta_{\varepsilon}^{\mathrm{Dir}} \Phi=f+\sum_{F \in \mathscr{F}_{\text {int }}} g_{F} \otimes \delta_{F}
$$

(i) If for any corner $\boldsymbol{c}$ and any edge $\boldsymbol{e}$

$$
\Lambda_{\varepsilon}^{\operatorname{Dir}}\left(\Gamma_{c}\right) \cap(-1 / 2, s-1 / 2]=\emptyset \quad \text { and } \quad \Lambda_{\varepsilon}^{\operatorname{Dir}}\left(\Gamma_{e}\right) \cap(0, s]=\emptyset,
$$

then $\Phi$ belongs to $\mathrm{PH}^{s+1}(\Omega, \mathscr{P})$.

(ii) If for any corner $\boldsymbol{c}$ and any edge $\boldsymbol{e}$

$$
\Lambda_{\varepsilon}^{\operatorname{Dir}}\left(\Gamma_{c}\right) \not \supset s-\frac{1}{2} \quad \text { and } \quad \Lambda_{\varepsilon}^{\operatorname{Dir}}\left(\Gamma_{e}\right) \not \ngtr s,
$$

then $\Phi$ admits a splitting $\Phi_{0}+\Phi_{1}$ into a regular part $\Phi_{1} \in P H^{s+1}(\Omega, \mathscr{P})$ and a singular part $\Phi_{0} \in H^{1}(\Omega)$ generated by the spaces $Z_{\text {Dir }}^{\lambda}\left(\Gamma_{c}, \varepsilon\right)$ and $Z_{\text {Dir }}^{\lambda}\left(\Gamma_{e}, \varepsilon\right)$ for $\lambda$ in $\Lambda_{\varepsilon}^{\operatorname{Dir}}\left(\Gamma_{c}\right) \cap(-1 / 2, s-1 / 2)$ and $\Lambda_{\varepsilon}^{\operatorname{Dir}}\left(\Gamma_{e}\right) \cap(0, s)$ respectively. In particular, if $s \leq 1, \Delta_{\varepsilon}^{\mathrm{Dir}} \Phi_{0}=f_{0}$ with $f_{0} \in P H^{s-1}(\Omega, \mathscr{P})$.

For $c$ in the set $\mathscr{C}$ of corners of $(\Omega, \mathscr{P})$ and $\lambda \in \Lambda_{\varepsilon}^{\operatorname{Dir}}\left(\Gamma_{c}\right)$, let $\Psi_{c}^{\lambda, p}$ be a basis of $Z_{\mathrm{Dir}}^{\lambda}\left(\Gamma_{c}, \varepsilon\right)$ and denote by $\Phi_{c}^{\lambda, p}$ the function defined as

$$
\Phi_{c}^{\lambda, p}(x)=\chi_{c}\left(\rho_{c}\right) \Psi_{c}^{\lambda, p}\left(\rho_{c}, \vartheta_{c}\right)
$$

with a smooth cut-off function $\chi_{c}$ equal to 1 in a neighborhood of 0 and $\left(\rho_{c}, \vartheta_{c}\right)$ the polar coordinates associated with $\boldsymbol{c}$.

Similarly, for $\boldsymbol{e}$ in the set $\mathscr{E}$ of edges of $(\Omega, \mathscr{P})$ and $\lambda \in \Lambda_{\varepsilon}^{\operatorname{Dir}}\left(\Gamma_{\boldsymbol{e}}\right)$, let $\Psi_{e}^{\lambda, p}$ be a basis of $Z_{\text {Dir }}^{\lambda}\left(\Gamma_{e}, \varepsilon\right)$ and denote by $\Phi_{e}^{\lambda, p}$ the function defined as

$$
\Phi_{e}^{\lambda, p}(x)=\chi_{e}\left(\rho_{e}\right) \Psi_{e}^{\lambda, p}\left(\rho_{e}, \vartheta_{e}\right), \quad \text { with } \quad \rho_{e}=\frac{r_{e}}{d_{e}}
$$


where $\chi_{e}$ is a smooth cut-off function equal to 1 in a neighborhood of $0, d_{e}$ a smooth function on the closed edge $\overline{\boldsymbol{e}}$, which is equivalent to the distance to the endpoints of $\boldsymbol{e}$ and $\left(r_{\boldsymbol{e}}, \theta_{\boldsymbol{e}}, z_{\boldsymbol{e}}\right)$ the cylindrical coordinates associated with $e$.

In order to give a precise statement, we still need weighted Sobolev spaces for the edge singularity coefficients and a smoothing operator, exactly as in [8]: let for $m \in \mathbb{N}$ and $\eta \in \mathbb{R}, \mathbb{V}_{\eta}^{m}(\boldsymbol{e})$ be defined as

$$
\mathbb{V}_{\eta}^{m}(\boldsymbol{e})=\left\{\gamma \in L^{2}(\boldsymbol{e}) \mid \quad\left(d_{\boldsymbol{e}}\right)^{\eta+k} \partial_{z_{e}}^{k} \gamma \in L^{2}(\boldsymbol{e}), \quad k=0,1, \ldots, m\right\}
$$

and by interpolation for non-integer $m$. The smoothing operator $\mathscr{K}[\cdot]$ acts like a lifting of functions on $e$ into $\Omega$ : in order to define it, we introduce the stretched variable

$$
\tilde{z}_{e}=\int_{0}^{z_{e}} \frac{1}{d_{e}(z)} \mathrm{d} z
$$

where $z=0$ corresponds to an interior point of $e$. The change of variable $z_{e} \mapsto \tilde{z}_{e}$ is one to one $\boldsymbol{e} \rightarrow \mathbb{R}$ and for any function $\gamma$ defined on $\boldsymbol{e}$, we set $\tilde{\gamma}\left(\tilde{z}_{\boldsymbol{e}}\right)=\gamma\left(z_{\boldsymbol{e}}\right)$. Then $\mathscr{K}[\gamma]\left(\rho_{\boldsymbol{e}}, \theta_{\boldsymbol{e}}, z_{\boldsymbol{e}}\right)$ is the convolution operator with respect to $\tilde{z}_{e}$ :

$$
\mathscr{K}[\gamma]\left(\rho_{e}, \theta_{e}, z_{e}\right)=\int_{\mathbb{R}} \frac{1}{\rho_{e}} \varphi\left(\frac{t}{\rho_{e}}\right) \tilde{\gamma}\left(t-\tilde{z}_{e}\right) \mathrm{d} t \quad \text { with } \rho_{e}=\frac{r_{e}}{d_{e}},
$$

where $\varphi$ is a smooth function in $\mathscr{S}(\mathbb{R})$ such that $\int_{\mathbb{R}} \varphi=1$.

Proposition 4.2. Let the assumptions of (ii) in Theorem 4.1 be satisfied. We assume moreover that for any edge $\boldsymbol{e}$, the set $\Lambda_{\varepsilon}^{\operatorname{Dir}}\left(\Gamma_{e}\right) \cap[0, s]$ is contained in an interval of length $<1$ (this is a technical assumption to avoid the "shadows" of the main singularities $\left.\Psi_{e}^{\lambda, p}\right)$. Then the singular part $\Phi_{0}$ has the expansion

$$
\Phi_{0}=\sum_{c \in \mathscr{C}} \sum_{\lambda \in[-1 / 2, s-1 / 2]} \sum_{p} \gamma_{c}^{\lambda, p} \Phi_{c}^{\lambda, p}+\sum_{e \in \mathscr{E}} \sum_{\lambda \in[0, s]} \sum_{p} \mathscr{K}\left[\gamma_{e}^{\lambda, p}\right] \Phi_{e}^{\lambda, p}
$$

with the coefficients $\gamma_{c}^{\lambda, p}$ in $\mathbb{R}$ and $\gamma_{e}^{\lambda, p}$ in $\mathbb{V}_{-s}^{s-\lambda}(\boldsymbol{e})$. The sums extend over $\lambda$ in $[-1 / 2, s-1 / 2] \cap \Lambda_{\varepsilon}^{\operatorname{Dir}}\left(\Gamma_{c}\right)$ and $[0, s] \cap \Lambda_{\varepsilon}^{\operatorname{Dir}}\left(\Gamma_{e}\right)$, respectively.

\section{MAXWELl INTERFACE CORNER SINGUlARITIES}

For shortness, we here describe the corner singularities of problem (1.5) (the singularities of problem (1.6) are obtained similarly by exchanging Dir, $\varepsilon$ and Neu, $\mu$ respectively). We further assume that $\Omega$ is simply connected.

We fix a corner $\boldsymbol{c}$ of $(\Omega, \mathscr{P})$ and drop the index $\boldsymbol{c}$ in the notations. At this stage, we look for solutions of the homogeneous Maxwell interface systems in the spaces of pseudo-homogeneous functions

$$
\boldsymbol{S}_{N}^{\lambda}(\Gamma, \varepsilon)=\left\{\boldsymbol{u} \in \boldsymbol{X}_{N}^{\mathrm{loc}}\left(\Gamma^{*}, \varepsilon\right) \mid \operatorname{div}(\varepsilon \boldsymbol{u}) \in H_{\mathrm{loc}}^{1}\left(\Gamma^{*}\right), \quad \boldsymbol{u}(x)=\rho^{\lambda} \sum_{q=0}^{Q} \log ^{q} \rho \boldsymbol{U}_{q}(\vartheta)\right\},
$$

where $\boldsymbol{u} \in \boldsymbol{X}_{N}^{\text {loc }}\left(\Gamma^{*}, \varepsilon\right)$ means that $\boldsymbol{u} \in \boldsymbol{X}_{N}^{\text {loc }}(\Gamma \cap \mathcal{V}, \varepsilon)$, for all bounded open sets $\mathcal{V}$ such that $\boldsymbol{c} \notin \overline{\mathcal{V}}$ : this space requires exactly the angular regularity corresponding to the effective regularity of the variational solution (in particular, for the condition $\operatorname{div}(\varepsilon \boldsymbol{u}) \in H_{\mathrm{loc}}^{1}\left(\Gamma^{*}\right)$, we rely on Th. 1.1). In other words, we have to find the non-polynomial solutions of the system

$$
\begin{cases}\operatorname{curl}\left(\mu^{-1} \operatorname{curl} \boldsymbol{u}\right)-\varepsilon \operatorname{grad} \operatorname{div}(\varepsilon \boldsymbol{u})=\boldsymbol{f} & \text { in } \Gamma \\ \operatorname{div}(\varepsilon \boldsymbol{u})=0 & \text { on } \partial \Gamma \\ \boldsymbol{u} \in \boldsymbol{S}_{N}^{\lambda}(\Gamma, \varepsilon) & \end{cases}
$$


when $\boldsymbol{f}$ is a homogeneous polynomial of degree $\lambda-2$ (thus it is zero if $\lambda \notin\{2,3, \ldots\}$ ). The corresponding $\lambda$ are the Maxwell (Dirichlet) corner exponents.

Like in [8], this problem is split into three subproblems by introducing the auxiliary unknowns

$$
\psi=\mu^{-1} \operatorname{curl} \boldsymbol{u} \quad \text { and } \quad q=\operatorname{div}(\varepsilon \boldsymbol{u}) .
$$

Using also the space $\boldsymbol{S}_{T}^{\lambda}(\Gamma, \mu)$ defined like $\boldsymbol{S}_{N}^{\lambda}(\Gamma, \varepsilon)$ and the space $S_{0}^{\lambda}(\Gamma)$ introduced in Section 4.1, we then see that for $\lambda \notin\{2,3, \ldots\}$, problem (5.1) is equivalent to finding non-polynomial solutions to the system

$$
\begin{aligned}
& -\Delta_{\varepsilon}^{\mathrm{Dir}} q=0 \text { in } \Gamma \quad \text { with } q \in S_{0}^{\lambda-1}(\Gamma) . \\
& \operatorname{curl} \psi=\varepsilon \operatorname{grad} q \quad \text { and } \quad \operatorname{div}(\mu \psi)=0 \text { in } \Gamma \quad \text { with } \psi \in \boldsymbol{S}_{T}^{\lambda-1}(\Gamma, \mu) . \\
& \operatorname{curl} \boldsymbol{u}=\mu \psi \quad \text { and } \quad \operatorname{div}(\varepsilon \boldsymbol{u})=q \text { in } \Gamma \quad \text { with } \quad \boldsymbol{u} \in \boldsymbol{S}_{N}^{\lambda}(\Gamma, \varepsilon) \text {. }
\end{aligned}
$$

Thus, the solutions of the system (5.2) belong to one of the three types:

Type 1. $q=0, \psi=0$ and $\boldsymbol{u}$ general non-zero solution of (5.2c).

Type 2. $q=0, \psi$ general non-zero solution of $(5.2 \mathrm{~b})$ and $\boldsymbol{u}$ particular solution of (5.2c).

Type 3. $q$ general non-zero solution of (5.2a), $\psi$ particular solution of (5.2b) and $\boldsymbol{u}$ particular solution of (5.2c).

These three types of Maxwell singularities are now described with the help of the corner singularities of $\Delta_{\varepsilon}^{\text {Dir }}$ and $\Delta_{\mu}^{\mathrm{Neu}}$. The singularities of type 1 are treated exactly as in Lemma 7.4 of [8].

Lemma 5.1. We assume that $\lambda \neq-1$. Then (i) is equivalent to (ii):

(i) $\boldsymbol{u} \in \boldsymbol{S}_{N}^{\lambda}(\Gamma, \varepsilon)$ is a solution of (5.2) of type 1,

(ii) $\lambda+1$ belongs to $\Lambda_{\varepsilon}^{\operatorname{Dir}}(\Gamma)$ and $\boldsymbol{u}=\operatorname{grad} \Phi$ where $\Phi$ belongs to $Z_{\operatorname{Dir}}^{\lambda+1}(\Gamma, \varepsilon)$.

For singularities of types 2 and 3 , the jumps of the product $\varepsilon \mu$ through the interfaces require a special attention.

Lemma 5.2. We assume that $\lambda$ is not an integer. Then (i) is equivalent to (ii):

(i) $\boldsymbol{u} \in \boldsymbol{S}_{N}^{\lambda}(\Gamma, \varepsilon)$ is a solution of (5.2) of type 2 ,

(ii) $\lambda$ belongs to $\Lambda_{\mu}^{\mathrm{Neu}}(\Gamma)$ and $\operatorname{curl} \boldsymbol{u}=\mu \operatorname{grad} \Psi$ where $\Psi$ belongs to $Z_{\mathrm{Neu}}^{\lambda}(\Gamma, \mu)$. In that case, a representative of type 2 is given by

$$
\boldsymbol{u}=\frac{1}{\lambda+1}\left(\mu(\operatorname{grad} \Psi \times \boldsymbol{x})+\operatorname{grad} r_{N}\right)
$$

where $r_{N} \in S^{\lambda+1}(\Gamma)$ is a solution of

$$
\Delta_{\varepsilon}^{\mathrm{Dir}} r_{N}=\left.\sum_{F \in \mathscr{F}_{\mathrm{int}, c}}[\varepsilon \mu]_{F}((\operatorname{grad} \Psi \times \boldsymbol{n}) \cdot \boldsymbol{x})\right|_{F} \otimes \delta_{F}
$$

Proof. We simply need to investigate the non-zero solutions $(\psi, \boldsymbol{u})$ of $(5.2)$ of type 2. First a non-zero $\Psi$ in $Z_{\text {Neu }}^{\lambda}(\Gamma, \mu)$ yields a non-zero requested $\psi=\operatorname{grad} \Psi$ (because $\lambda \neq 0$ ). It then remains to find $\boldsymbol{u} \in \boldsymbol{S}_{N}^{\lambda}(\Gamma, \varepsilon)$ such that

$$
\operatorname{curl} \boldsymbol{u}=\mu \psi \text { and } \operatorname{div}(\varepsilon \boldsymbol{u})=0 \text { in } \Gamma \text {. }
$$


We are then looking for $\boldsymbol{u}$ of the form (5.3). In that case, we have

$$
\begin{aligned}
(\lambda+1) \operatorname{curl} \boldsymbol{u} & =\operatorname{curl}(\mu \operatorname{grad} \Psi \times \boldsymbol{x}) \\
& =\boldsymbol{x} \cdot \operatorname{grad}(\mu \psi)-\mu \psi \cdot \operatorname{grad} \boldsymbol{x}+\mu \psi \operatorname{div} \boldsymbol{x}-\boldsymbol{x} \operatorname{div}(\mu \psi),
\end{aligned}
$$

due to the identity $(7.5 \mathrm{~b})$ of [8]. This yields

$$
\operatorname{curl} \boldsymbol{u}=\mu \psi,
$$

because $\psi$ is homogeneous, $\operatorname{div}(\mu \psi)=0$ and one can show that

$$
\boldsymbol{x} \cdot \operatorname{grad}(\mu \psi)=\mu \boldsymbol{x} \cdot \operatorname{grad} \psi
$$

in the distributional sense.

On the other hand, the conditions $\operatorname{div}(\varepsilon \boldsymbol{u})=0$ and $\boldsymbol{u} \times \boldsymbol{n}=0$ on $\partial \Gamma$ will hold if (5.4) holds since

$$
\operatorname{div}(\varepsilon \mu(\operatorname{grad} \Psi \times \boldsymbol{x}))=\sum_{F \in \mathscr{F}_{\text {int }, c}} g_{F} \otimes \delta_{F},
$$

where

$$
g_{F}=[\varepsilon \mu(\operatorname{grad} \Psi \times \boldsymbol{x}) \cdot \boldsymbol{n}]_{F}=-[\varepsilon \mu(\operatorname{grad} \Psi \times \boldsymbol{n}) \cdot \boldsymbol{x}]_{F}=-\left.[\varepsilon \mu]_{F}((\operatorname{grad} \Psi \times \boldsymbol{n}) \cdot \boldsymbol{x})\right|_{F},
$$

since grad $\Psi \times \boldsymbol{n}$ has no jump across the interfaces. By Theorem 4.14 of [18], problem (5.4) has a solution $r_{N} \in S^{\lambda+1}(\Gamma)$ (in view of that theorem, one sees that $r_{N}$ is homogeneous if $\lambda+1 \notin \Lambda_{\varepsilon}^{\operatorname{Dir}}(\Gamma)$ and has the form $r_{N}=r_{0}+r_{1} \log \rho$, with homogeneous $r_{0}$ and $r_{1}$ if not).

This guarantees the existence of $\boldsymbol{u}$.

Similarly, we can show:

Lemma 5.3. We assume that $\lambda$ is not integer. Then (i) is equivalent to (ii):

(i) $\boldsymbol{u} \in \boldsymbol{S}_{N}^{\lambda}(\Gamma, \varepsilon)$ is a solution of (5.2) of type 3 ,

(ii) $\lambda-1$ belongs to $\Lambda_{\varepsilon}^{\operatorname{Dir}}(\Gamma)$ and $\operatorname{div}(\varepsilon \boldsymbol{u})=q$ where $q$ belongs to $Z_{\operatorname{Dir}}^{\lambda-1}(\Gamma, \varepsilon)$.

To each $q \in Z_{\text {Dir }}^{\lambda-1}(\Gamma, \varepsilon)$, a representative of type 3 is given by

$$
\psi=\frac{1}{\lambda}\left(\varepsilon(\operatorname{grad} q \times \boldsymbol{x})+\operatorname{grad} r_{T}\right),
$$

where $r_{T} \in S^{\lambda}(\Gamma)$ is a solution of

$$
\Delta_{\mu}^{\mathrm{Neu}} r_{T}=\left.\sum_{F \in \mathscr{F}_{\text {int }, c}}[\varepsilon \mu]_{F}((\operatorname{grad} q \times \boldsymbol{n}) \cdot \boldsymbol{x})\right|_{F} \otimes \delta_{F},
$$

and, if $\lambda \notin \Lambda_{\mu}^{\mathrm{Neu}}(\Gamma)$, by

where $r_{N} \in S^{\lambda+1}(\Gamma)$ is a solution of

$$
\boldsymbol{u}=\frac{1}{\lambda+1}\left(\mu(\psi \times \boldsymbol{x})+\operatorname{grad} r_{N}\right)
$$

$$
\Delta_{\varepsilon}^{\mathrm{Dir}} r_{N}=\left.\sum_{F \in \mathscr{F}_{\text {int }, c}}[\varepsilon \mu]_{F}((\psi \times \boldsymbol{n}) \cdot \boldsymbol{x})\right|_{F} \otimes \delta_{F}+\left(\left(1-\varepsilon^{2} \mu\right) \lambda+1+\varepsilon^{2} \mu\right) q .
$$


TABLE 1

\begin{tabular}{|c|c|c|c|c|c|c|}
\hline Type & $\lambda$ & $>$ & Generator & $u$ & $\psi$ & $q$ \\
\hline 1 & $\lambda+1 \in \Lambda_{\varepsilon}^{\mathrm{Dir}}(\Gamma)$ & -1 & $\Phi \in Z_{\text {Dir }}^{\lambda+1}(\Gamma, \varepsilon)$ & $\operatorname{grad} \Phi$ & 0 & 0 \\
\hline 2 & $\lambda \in \Lambda_{\mu}^{\mathrm{Neu}}(\Gamma)$ & 0 & $\Psi \in Z_{\mathrm{Neu}}^{\lambda}(\Gamma, \mu)$ & $c f$. Lem. 5.2 & $\operatorname{grad} \Psi$ & 0 \\
\hline 3 & $\lambda-1 \in \Lambda_{\varepsilon}^{\mathrm{Dir}}(\Gamma)$ & 1 & $q \in Z_{\mathrm{Dir}}^{\lambda-1}(\Gamma, \varepsilon)$ & $c f$. Lem. 5.3 & $c f$. Lem. 5.3 & $q$ \\
\hline
\end{tabular}

It remains to investigate the singularities of type 1 for $\lambda=-1$ and of type 2 for $\lambda=0$.

\section{Lemma 5.4.}

(i) There is no singularity of type 1 for $\lambda=-1$.

(ii) There is no singularity of type 2 for $\lambda=0$.

Proof. Since $\Gamma$ is simply connected, the first assertion is proved exactly as in Lemma 7.8 of [8]: we obtain that if $\boldsymbol{u}$ belongs to $\boldsymbol{S}_{N}^{-1}(\Gamma, \varepsilon)\left[\right.$ resp. $\left.\boldsymbol{S}_{T}^{-1}(\Gamma, \mu)\right]$ and satisfies $\operatorname{curl} \boldsymbol{u}=0$ and $\operatorname{div}(\varepsilon \boldsymbol{u})=0[\operatorname{resp} . \operatorname{div}(\mu \boldsymbol{u})=0]$, then $\boldsymbol{u}=0$.

For the second one, we simply remark that if $\boldsymbol{u}$ is a singularity of type 2 in $\boldsymbol{S}_{N}^{0}(\Gamma, \varepsilon)$, then

$$
\psi=\mu^{-1} \operatorname{curl} u \in S_{T}^{-1}(\Gamma, \mu)
$$

is a solution of type 1 for magnetic boundary conditions. Therefore the first assertion yields $\psi=0$ and the conclusion follows.

Remark 5.5. The case $\Gamma$ not simply connected can be treated as in $[8]$ and would yield topological singular exponents. This case was avoided for brevity and is left to the reader. For other problems with multiplyconnected domains, see also [1,11].

Among the singular exponents obtained before, we select the subset $\Lambda_{N}(\Gamma)$ of $\lambda$ satisfying $\lambda>-3 / 2$ such that there exists a non-zero $\boldsymbol{u} \in \boldsymbol{S}_{N}^{\lambda}(\Gamma, \varepsilon)$ solution of (5.1) and satisfying ( $c f$. Th. 1.1)

$$
\chi \boldsymbol{u} \in \boldsymbol{X}_{N}(\Gamma, \varepsilon), \operatorname{div}(\chi \varepsilon \boldsymbol{u}) \in H^{1}(\Gamma),
$$

with a cut-off function $\chi$ which is equal to 1 in a neighborhood of the corner $\boldsymbol{c}$. We examine the effect of this condition on the three types of singularities.

Type 1. $\lambda+1$ belongs to $\Lambda_{\varepsilon}^{\operatorname{Dir}}(\Gamma)$. Since $\Lambda_{\varepsilon}^{\operatorname{Dir}}(\Gamma) \cap[-1,0]$ is empty, with Lemma 5.4 we get the condition $\lambda>-1$. Type 2. $\lambda \in \Lambda_{\mu}^{\mathrm{Neu}}(\Gamma)$. Since $\operatorname{curl}(\chi \boldsymbol{u})=\chi \operatorname{curl} \boldsymbol{u}+\operatorname{grad} \chi \times \boldsymbol{u}$ has to be in $L^{2}(\Gamma)^{3}$, we have the condition $\lambda>-1 / 2$. With Lemma 5.4 , this yields $\lambda>0$, because the set $\Lambda_{\mu}^{\mathrm{Neu}}(\Gamma) \cap[-1,0]$ is empty.

Type 3. Here $\lambda-1$ belongs to $\Lambda_{\varepsilon}^{\operatorname{Dir}}(\Gamma)$. Thus condition $\operatorname{div}(\chi \varepsilon \boldsymbol{u})$ in $H^{1}(\Gamma)$ implies that $\chi q$ belongs to $H^{1}(\Gamma)$, thus $\lambda-1>-1 / 2$, whence $\lambda-1>0$, or equivalently $\lambda>1$.

We summarize the above results in Table 1.

Going back to the primitive Maxwell equation (1.1), we see that for a regular current density $\boldsymbol{J}, \operatorname{div}(\varepsilon \boldsymbol{E})$ and $\operatorname{div}(\mu \boldsymbol{H})$ are regular too, thus only the singularities of types 1 and 2 can occur and they exchange each 
TABLE 2

\begin{tabular}{|c|c|c|c|c|}
\hline Type & Generator & $\lambda \in$ & $\boldsymbol{E}$ & $\boldsymbol{H}$ \\
\hline \hline Elec. & $\Phi \in Z_{\mathrm{Dir}, \varepsilon}^{\lambda}$ & $\Lambda_{\varepsilon}^{\text {Dir }}$ & $\operatorname{grad} \Phi$ & $-\kappa\left(\varepsilon \operatorname{grad} \Phi \times \boldsymbol{x}+\operatorname{grad} r_{T}\right)$ \\
\hline Magn. & $\Psi \in Z_{\mathrm{Neu}, \mu}^{\lambda}$ & $\Lambda_{\mu}^{\mathrm{Neu}}$ & $\kappa\left(\mu \operatorname{grad} \Psi \times \boldsymbol{x}+\operatorname{grad} r_{N}\right)$ & $\operatorname{grad} \Psi$ \\
\hline
\end{tabular}

other between the electric and magnetic fields (here $\lambda$ denotes the degree of homogeneity of the generator and is either the degree of $\boldsymbol{E}$ or $\boldsymbol{H}$ and $\kappa=\frac{i \omega}{\lambda+1}$ ):

Table 2 gives the principal parts of the singularities, indeed from (1.5) and (1.6) we see that the operators are not homogeneous and therefore the singularities have an asymptotic expansion $[9,13]$.

\section{MAXWELL INTERFACE EDGE SINGULARITIES}

In this section, our aim is to describe shortly the edge singularities of problem (1.5). Fix one edge $e$ of $(\Omega, \mathscr{P})$, see Section 4.2 for the associated definitions (we drop here the index $\boldsymbol{e})$. Let $\lambda \in \mathbb{C}$. According to the general rule [9], we search for (non-polynomial) solutions $\boldsymbol{u} \in \boldsymbol{S}_{N}^{\lambda}(\Gamma \times \mathbb{R}, \varepsilon)$ independent of $z$ of the system

$$
\operatorname{curl}\left(\mu^{-1} \operatorname{curl} \boldsymbol{u}\right)-\varepsilon \operatorname{grad} \operatorname{div}(\varepsilon \boldsymbol{u})=\boldsymbol{f} \text { in } \Gamma \times \mathbb{R},
$$

with $\boldsymbol{f}$ independent of $z$ and polynomial in the $\boldsymbol{y}$ variable. The corresponding $\lambda$ are the Maxwell (Dirichlet) edge exponents. Let now $(\boldsymbol{v}, w)$ be the decomposition of the field $\boldsymbol{u}$ in the system of Cartesian coordinates $(\boldsymbol{y}, z)$. Then this system is split into 2 two-dimensional independent problems in the sector $\Gamma$ :

$$
\begin{cases}\operatorname{curl}\left(\mu^{-1} \operatorname{curl} \boldsymbol{v}\right)-\varepsilon \operatorname{grad} \operatorname{div}(\varepsilon \boldsymbol{v})=\boldsymbol{f} & \text { in } \Gamma, \boldsymbol{f} \text { polynomial, } \\ \boldsymbol{v} \times \boldsymbol{n}=0 \text { and } \operatorname{div}(\varepsilon \boldsymbol{v})=0 & \text { on } \partial \Gamma, \\ \boldsymbol{v} \in \boldsymbol{S}_{N}^{\lambda}(\Gamma, \varepsilon) & \end{cases}
$$

and

$$
\begin{cases}-\operatorname{div}\left(\mu^{-1} \operatorname{grad} w\right)=f & \text { in } \Gamma, f \text { polynomial } \\ w=0 & \text { on } \partial \Gamma \\ w \in S^{\lambda}(\Gamma) & \end{cases}
$$

The problem (6.1) is simply the problem attached to two-dimensional Maxwell equations in a polygonal domain, and (6.2) is the transmission Dirichlet problem whose set $\Lambda_{\mu^{-1}}^{\operatorname{Dir}}(\Gamma)$ of singularities is well known.

For the two-dimensional "Maxwell-type" problem (6.1), as in 3D, we introduce two auxiliary (scalar) variables

$$
\psi=\mu^{-1} \operatorname{curl} \boldsymbol{v} \quad \text { and } \quad q=\operatorname{div}(\varepsilon \boldsymbol{v}) .
$$

Then for $\lambda \notin \mathbb{N}$, we get the equivalent system

$$
\begin{array}{lrl}
-\Delta_{\varepsilon}^{\mathrm{Dir}} q=\operatorname{div} \boldsymbol{f} \text { in } \Gamma & \text { with } & q \in S_{0}^{\lambda-1}(\Gamma) . \\
\operatorname{curl} \psi=\varepsilon \operatorname{grad} q \text { in } \Gamma & \text { with } & \psi \in S^{\lambda-1}(\Gamma) . \\
\operatorname{curl} \boldsymbol{v}=\mu \psi, \operatorname{div}(\varepsilon \boldsymbol{v})=q \text { in } \Gamma & \text { with } \boldsymbol{u} \in \boldsymbol{S}_{N}^{\lambda}(\Gamma, \varepsilon) .
\end{array}
$$


If $\lambda$ is not a positive integer, as in the previous section, this system (6.4) is reduced to a homogeneous one and the solutions split into singularities of types 1,2 and 3. As in [8], the singularities of type 2 do not exist [they appear in fact as singularities of the problem (6.2)], while the singularities of types 1 and 3 are obtained like in Section 5 in relation with the edge exponents of $\Delta_{\varepsilon}^{\text {Dir }}$.

If $\lambda$ is a positive integer, as in Section 4.2, we can check that the spaces of homogeneous polynomials associated with the right hand sides and with the solutions have the same dimension. Thus the Maxwell edge exponents are the $\lambda \in \mathbb{C}$ such that the system (6.4) has non-trivial solutions.

In view of (4.7), we can state:

Lemma 6.1. The set of the edge exponents associated with the edge $\boldsymbol{e}$ is

$$
\left\{\lambda \in \mathbb{R} \mid \lambda-1 \text { or } \lambda+1 \text { belongs to } \Lambda_{\varepsilon}^{\operatorname{Dir}}(\Gamma)\right\} \cup \Lambda_{\mu^{-1}}^{\text {Dir }}(\Gamma) .
$$

If $\lambda \notin \mathbb{N}^{*}$, the corresponding singular functions $\boldsymbol{u}=(\boldsymbol{v}, w)$ are as follows:

(i) If $\lambda+1 \in \Lambda_{\varepsilon}^{\operatorname{Dir}}(\Gamma)$, then $w=0$ and $\boldsymbol{v}$ is a Maxwell singularity of type 1, given by

$$
\boldsymbol{v}=\operatorname{grad}\left(r^{\lambda+1} \varphi(\theta)\right)
$$

when $\varphi$ is an eigenvector of $L_{\varepsilon, \boldsymbol{e}}^{\mathrm{Dir}}$ associated with the eigenvalue $(\lambda+1)^{2}$.

(ii) If $\lambda \in \Lambda_{\mu^{-1}}^{\mathrm{Dir}}(\Gamma)$, then $\boldsymbol{v}=0$ and $w$ is a singularity associated with $\Delta_{\mu^{-1}}^{\mathrm{Dir}}$ :

$$
w=r^{\lambda} \varphi(\theta)
$$

when $\varphi$ is an eigenvector of $L_{\mu^{-1}, e}^{\mathrm{Dir}}$ associated with the eigenvalue $\lambda^{2}$.

(iii) If $\lambda-1 \in \Lambda_{\varepsilon}^{\operatorname{Dir}}(\Gamma)$, then $w=0$ and $\boldsymbol{v}$ is a Maxwell singularity of type 3 .

The singularities in point (ii) of the lemma are, in fact, closely related to the type 2 corner singularities. This is seen from the following result.

Lemma 6.2. We have the identity between the sets of Laplace edge exponents

$$
\Lambda_{\mu^{-1}}^{\mathrm{Dir}}(\Gamma)=\Lambda_{\mu}^{\mathrm{Neu}}(\Gamma)
$$

and more precisely we have the equivalence between the singular functions

$$
r^{\lambda} \psi(\theta) \in Z_{\text {Neu }}^{\lambda}(\Gamma, \mu) \quad \Longleftrightarrow \mu r^{\lambda} \partial_{\theta} \psi \in Z_{\text {Dir }}^{\lambda}\left(\Gamma, \mu^{-1}\right) .
$$

Proof. The proof uses the fact that in dimension 2 the passage to the conjugate harmonic functions interchanges tangential and normal derivatives. This implies that a singular function $\Psi$ belongs to $Z_{\mathrm{Neu}}^{\lambda}(\Gamma, \mu)$ if and only if $\mu \widetilde{\Psi} \in Z_{\text {Dir }}^{\lambda}\left(\Gamma, \mu^{-1}\right)$, where on each sector $\Gamma_{j}, \widetilde{\Psi}$ is the harmonic conjugate of $\Psi$. Since for our homogeneous functions, $\widetilde{\Psi}$ can be expressed by the angular derivative, we can make this idea more precise as follows: let $\Delta_{\mu}^{\mathrm{Neu}}\left(r^{\lambda} \psi(\theta)\right)=0$ in $\Gamma$. This means that

$$
\partial_{\theta} \mu \partial_{\theta} \psi+\lambda^{2} \mu \psi=0, \text { thus } \mu^{-1} \partial_{\theta}\left(\mu \partial_{\theta} \psi\right)+\lambda^{2} \psi=0 .
$$

Setting $\varphi=\mu \partial_{\theta} \psi$, the interface conditions $[\psi]=0$ and $\left[\mu \partial_{\theta} \psi\right]=0$ imply therefore that $[\varphi]=0$ and $\left[\mu^{-1} \partial_{\theta} \varphi\right]=0$. Whence the lemma.

As before, we have to consider the subset of the edge exponents $\lambda$ satisfying $\lambda>-1$ such that there exists a non-zero $\boldsymbol{u} \in \boldsymbol{S}_{N}^{\lambda}(\Gamma \times \mathbb{R}, \varepsilon)$ independent of the variable $z$, solution of the homogeneous system (6.1-6.2) and satisfying

$$
\operatorname{curl}(\chi \boldsymbol{u}) \in L^{2}(\Gamma)^{3}, \operatorname{div}_{y}(\chi \varepsilon \boldsymbol{u}) \in H^{1}(\Gamma),
$$


with $\chi$ a cut-off function which is equal to 1 in a neighborhood of the corner of $\Gamma$. The effect of this condition on each of the singularities (i), (ii) and (iii) in Lemma 6.1 is easily checked and can be summarized as follows:

(i) In this case $\lambda_{1}=\tilde{\lambda}-1$, with $\tilde{\lambda} \in \Lambda_{\varepsilon}^{\operatorname{Dir}}(\Gamma)$ and the condition is $\lambda_{1}>-1$.

(ii) In this case $\lambda_{2} \in \Lambda_{\mu^{-1}}^{\mathrm{Dir}}(\Gamma)$, thus $\lambda_{2}$ has to be positive.

(iii) In this case $\lambda_{3}=\tilde{\lambda}+1$, with $\tilde{\lambda} \in \Lambda_{\varepsilon}^{\operatorname{Dir}}(\Gamma)$, then the condition is $\lambda_{3}>1$.

\section{Conclusions}

\subsection{Regularity}

Taking advantage of the information about corner and edge exponents and singularities collected in Sections 4 to 6 and using Theorem 4.1 of [8] (which also hold in our setting with the natural adaptations due to the interfaces), we are now able to give regularity results.

As always, the regularity depends on the smallest corner and edge exponents. So, for any edge $\boldsymbol{e}$ in the set $\mathscr{E}$ of the edges of $(\Omega, \mathscr{P})$, we introduce the smallest exponent attached to $\Delta_{\varepsilon}^{\text {Dir }}$

$$
\lambda_{\varepsilon, \boldsymbol{e}}^{\mathrm{Dir}}=\sqrt{\nu}, \quad \text { with } \nu \text { the first eigenvalue of } L_{\varepsilon, \boldsymbol{e}}^{\mathrm{Dir}}
$$

and the smallest exponent attached to $\Delta_{\mu}^{\mathrm{Neu}}$

$$
\lambda_{\mu, e}^{\mathrm{Neu}}=\sqrt{\nu}, \quad \text { with } \nu \text { the first non-zero eigenvalue of } L_{\mu, \boldsymbol{e}}^{\mathrm{Neu}} .
$$

We have the following lower estimates for $\lambda_{\varepsilon, e}^{\text {Dir }}$ (and similar ones for $\lambda_{\mu, e}^{\mathrm{Neu}}$ ). Proofs are given in Section 8 .

(i) With $\rho_{\boldsymbol{e}}$ the quotient of the minimum of $\varepsilon$ by its maximum in the neighborhood of $\boldsymbol{e}$, a lower estimate of the Rayleigh quotient of $L_{\varepsilon, e}^{\text {Dir }}$ yields

$$
\lambda_{\varepsilon, \boldsymbol{e}}^{\mathrm{Dir}} \geq \rho_{e} \lambda_{1, \boldsymbol{e}}^{\mathrm{Dir}}
$$

(ii) If $\boldsymbol{e}$ is an external edge:

- For two subdomains in a convex angle $\lambda_{\varepsilon, e}^{\text {Dir }}>1 / 2$.

- For two subdomains in a non-convex angle $\lambda_{\varepsilon, e}^{\text {Dir }}>1 / 4,[19]$.

- For three subdomains (even in a convex angle) $\lambda_{\varepsilon, e}^{\text {Dir }}>0,[14]$.

(iii) If $\boldsymbol{e}$ is an internal edge:

- For two subdomains $\lambda_{\varepsilon, e}^{\mathrm{Dir}}>1 / 2$.

- For three subdomains $\lambda_{\varepsilon, e}^{\text {Dir }}>1 / 4$.

- For four subdomains $\lambda_{\varepsilon, e}^{\text {Dir }}>0$.

The estimates in (ii) and (iii) are generically optimal in the sense that there exist choices of $\Gamma$ and $\varepsilon$ so that $\lambda_{\varepsilon, e}^{\text {Dir }}$ is arbitrarily close to the lower bound.

Similarly, for any corner $\boldsymbol{c}$ in the set $\mathscr{C}$ of the corners of $(\Omega, \mathscr{P})$, we introduce the smallest exponent attached to $\Delta_{\varepsilon}^{\text {Dir }}$ (see Sect. 4.1)

$$
\lambda_{\varepsilon, c}^{\operatorname{Dir}}=\min \left(\Lambda_{\varepsilon, c}^{\operatorname{Dir}} \cap(-1 / 2, \infty)\right)
$$

and the smallest exponent attached to $\Delta_{\mu}^{\mathrm{Neu}}$

$$
\lambda_{\mu, c}^{\mathrm{Neu}}=\min \left(\Lambda_{\mu, c}^{\mathrm{Neu}} \cap(-1 / 2, \infty)\right) .
$$

In general $\lambda_{\varepsilon, c}^{\text {Dir }}$ is the minimum of 2 and of $-1 / 2+\sqrt{\nu+1 / 4}$, with $\nu$ the first eigenvalue of $L_{\varepsilon, c}^{\text {Dir }}$, and similarly for $\lambda_{\mu, c}^{\mathrm{Neu}}$. In any case, $\lambda_{\varepsilon, c}^{\mathrm{Dir}}$ and $\lambda_{\mu, c}^{\mathrm{Neu}}$ are $>0$ and satisfy a lower estimate like (7.1) by the exponents associated with one material in the same corner. 
Let now set

$$
\sigma_{\varepsilon}^{\mathrm{Dir}}=\min \left(\min _{e \in \mathscr{E}} \lambda_{\varepsilon, e}^{\mathrm{Dir}}, \min _{c \in \mathscr{C}} \lambda_{\varepsilon, c}^{\mathrm{Dir}}+\frac{1}{2}\right) \quad \text { and } \quad \sigma_{\mu}^{\mathrm{Neu}}=\min \left(\min _{e \in \mathscr{E}} \lambda_{\mu, e}^{\mathrm{Neu}}, \min _{c \in \mathscr{C}} \lambda_{\mu, c}^{\mathrm{Neu}}+\frac{1}{2}\right) .
$$

In fact, the regularity result (i) of Theorem 4.1 holds with any $s<\sigma_{\varepsilon}^{\text {Dir }}$ for the operator $\Delta_{\varepsilon}^{\text {Dir }}$ and with any $s<\sigma_{\mu}^{\mathrm{Neu}}$ for the operator $\Delta_{\mu}^{\mathrm{Neu}}$.

Theorem 7.1. Let $s \geq 1$ and $\boldsymbol{f} \in P_{\boldsymbol{H}^{s-1}}(\Omega, \mathscr{P})$. Let $\boldsymbol{u} \in \boldsymbol{X}_{N}(\Omega, \varepsilon)$ be the solution of problem (1.5). For any $\tau \in(0, s+1]$ such that

$$
\tau<\min \left\{\sigma_{\varepsilon}^{\mathrm{Dir}}, \sigma_{\mu}^{\mathrm{Neu}}+1\right\}
$$

$\boldsymbol{u}$ belongs to $P \boldsymbol{H}^{\tau}(\Omega, \mathscr{P})$.

Examples.

(i) If $\Omega$ contains only two subdomains, then $\boldsymbol{u} \in P \boldsymbol{H}^{\tau}(\Omega, \mathscr{P})$ for all $\tau \leq 1 / 4$.

(ii) If $\Omega$ is convex and has two subdomains, then $\boldsymbol{u} \in P \boldsymbol{H}^{\tau}(\Omega, \mathscr{P})$ for all $\tau \leq 1 / 2$.

(iii) If $\Omega$ is a parallelepiped divided into two subdomains separated by a plane parallel to two faces, then $\boldsymbol{u} \in P \boldsymbol{H}^{\tau}(\Omega, \mathscr{P})$ for all $\tau<2$.

But note that, as soon as three subdomains have an exterior common edge, or four subdomains have an interior common edge, the regularity of $\boldsymbol{u}$ can be arbitrarily low (near $L^{2}$ ). Such a situation occurs when the ratio $\rho_{\boldsymbol{e}}$ is very small.

\subsection{Singularities}

In this whole subsection $s \geq 1$, the data $\boldsymbol{f}$ belongs to $P \boldsymbol{H}^{s-1}(\Omega, \mathscr{P})$ and $\boldsymbol{u}$ is the solution of problem (1.5).

A. We assume that $s$ is such that there is no Maxwell Dirichlet corner exponent equal to $s-1 / 2$ and no Maxwell Dirichlet edge exponent equal to $s$. Then $\boldsymbol{u}$ can be split in $\boldsymbol{u}_{0}+\boldsymbol{u}_{1}$ where $\boldsymbol{u}_{1}$ belongs to $P \boldsymbol{H}^{s+1}(\Omega, \mathscr{P})$ and $\boldsymbol{u}_{0}$ is the sum of contributions of the corners and the edges. If we assume moreover like in Proposition (4.2), that for any edge $\boldsymbol{e}$, the set of the edge exponents $\in[-1, s]$ is contained in an interval of length $<1$, the function $\boldsymbol{u}_{0}$ has a structure like $\Phi_{0}$ in (4.12)

$$
\boldsymbol{u}_{0}=\sum_{\boldsymbol{c} \in \mathscr{C}} \sum_{\lambda \in\left[-\frac{3}{2}, s-\frac{1}{2}\right]} \sum_{p} \gamma_{\boldsymbol{c}}^{\lambda, p} \boldsymbol{u}_{c}^{\lambda, p}+\sum_{\boldsymbol{e} \in \mathscr{E}} \sum_{\lambda \in[-1, s]} \sum_{p} \mathscr{K}\left[\gamma_{e}^{\lambda, p}\right] \boldsymbol{u}_{e}^{\lambda, p}
$$

with $\boldsymbol{u}_{c}^{\lambda, p}$ and $\boldsymbol{u}_{e}^{\lambda, p}$ defined like (4.10) and (4.11) from bases $\boldsymbol{U}_{c}^{\lambda, p}$ and $\boldsymbol{U}_{e}^{\lambda, p}$ of non-polynomial solutions of problems (5.1) and (6.1-6.2). If $\boldsymbol{U}_{e}^{\lambda, p}$ has no logarithmic term, then the coefficient belongs to $\mathbb{V}_{-s}^{s-\lambda}(\boldsymbol{e})$. For non-integer $\lambda$, the functions $\boldsymbol{U}_{c}^{\lambda, p}$ and $\boldsymbol{U}_{e}^{\lambda, p}$ are described in Lemmas 5.1-6.1.

B. Let us fix $\sigma \in[0, s]$ such that for any edge $\boldsymbol{e}$, the set of the edge exponents belonging to $[-1, \sigma]$ is contained in an interval of length $<1$. Then for suitable coefficients $\gamma_{\boldsymbol{c}}^{\lambda, p} \in \mathbb{R}$ and $\gamma_{\boldsymbol{e}}^{\lambda, p} \in \mathbb{V}_{-\sigma}^{s-\lambda}(\boldsymbol{e})$ the difference

$$
\boldsymbol{u}-\left(\sum_{c \in \mathscr{C}} \sum_{\lambda \in\left[-\frac{3}{2}, \sigma-\frac{1}{2}\right]} \sum_{p} \gamma_{\boldsymbol{c}}^{\lambda, p} \boldsymbol{u}_{c}^{\lambda, p}+\sum_{\boldsymbol{e} \in \mathscr{E}} \sum_{\lambda \in[-1, \sigma]} \sum_{p} \mathscr{K}\left[\gamma_{\boldsymbol{e}}^{\lambda, p}\right] \boldsymbol{u}_{e}^{\lambda, p}\right)
$$

belongs to $P \boldsymbol{H}^{\sigma+1}(\Omega, \mathscr{P})$.

If we take $\sigma=0$, or more generally

$$
\sigma_{\varepsilon}^{\text {Dir }}-1<\sigma<\min \left(\sigma_{\varepsilon}^{\text {Dir }}, \sigma_{\mu}^{\text {Neu }}\right)
$$


then the corner and edge singularities of types 2 and 3 disappear, therefore it only gradients remain in the singular part, which can be written as (cf. Sect. 4.3)

$$
\begin{aligned}
\sum_{c \in \mathscr{C}} \sum_{\lambda \in\left[-\frac{3}{2}, \sigma-\frac{1}{2}\right]} \sum_{p} \gamma_{c}^{\lambda, p} \chi_{c}\left(\rho_{c}\right) \operatorname{grad} \Psi_{c}^{\lambda+1, p}\left(\rho_{c}, \vartheta_{c}\right) \\
+\sum_{e \in \mathscr{E}} \sum_{\lambda \in[-1, \sigma]} \sum_{p} \mathscr{K}\left[\gamma_{e}^{\lambda, p}\right] \chi_{e}\left(\rho_{e}\right) \operatorname{grad}_{e} \Psi_{e}^{\lambda+1, p}\left(\rho_{e}, \theta_{e}\right),
\end{aligned}
$$

with $\operatorname{grad}_{e}$ the gradient in the variable $\tilde{\boldsymbol{y}}_{\boldsymbol{e}}=\boldsymbol{y}_{e} / d_{e}$.

Remark 7.2. In the splitting (7.5), the singular generators can also be expressed as curls since for a homogeneous function $\Psi$ of degree $\lambda$ satisfying $\Delta_{\varepsilon}^{\text {Dir }} \Psi=0$, we have:

$$
\varepsilon(\lambda+1) \operatorname{grad} \Psi=\operatorname{curl}(\varepsilon \operatorname{grad} \Psi \times \boldsymbol{x})
$$

and

$$
\operatorname{grad}_{e}\left(\rho_{e}^{\lambda} \varphi\left(\theta_{e}\right)\right)=\operatorname{curl}_{e}\left(\rho_{e}^{\lambda} \psi\left(\theta_{e}\right)\right)
$$

when $\psi=-\frac{1}{\lambda} \varphi^{\prime}$ (recalling that $\varphi$ satisfies $\left(\varepsilon \varphi^{\prime}\right)^{\prime}=-\lambda^{2} \varepsilon \varphi$ ), with $\operatorname{curl}_{e}$ the two-dimensional vectorial curl in the $\tilde{\boldsymbol{y}}_{\boldsymbol{e}}$ plane, completed by a zero tangential component along the edge.

As in [8], we can write the singular part (7.5) as a gradient in a global way, because Lemmas 8.2 and 8.4 of [8] are (mainly) independent of the operator in consideration. Consequently, in connection with the splitting (4.12), we have

Theorem 7.3. Assume that $s \geq 1$, the data $\boldsymbol{f}$ belongs to $P \boldsymbol{H}^{s-1}(\Omega, \mathscr{P})$ and $\boldsymbol{u}$ is the solution of problem (1.5). Let $\sigma<s+1$ so that (7.4) holds. Then there exists $\Phi \in \stackrel{\circ}{H}^{1}(\Omega)$ satisfying $-\Delta_{\varepsilon}^{\mathrm{Dir}} \Phi \in P H^{\sigma}(\Omega, \mathscr{P})$ such that

$$
\boldsymbol{u}-\operatorname{grad} \Phi \in P \boldsymbol{H}^{\sigma+1}(\Omega, \mathscr{P}) .
$$

When $\sigma=0$, the above statement reduces to Theorem 3.5 (ii).

\section{APPENDIX}

In this section, we prove some lower estimates for the exponents of singularity for transmission problems for the Laplacian in dimension two. We have to consider the following situation:

$\Gamma$ is described in polar coordinates $(r, \theta)$ by $0<\theta<\omega(0<\omega<2 \pi)$ or by $0 \leq \theta<2 \pi(\omega=2 \pi)$. The interval $[0, \omega]$ is divided in $J$ subintervals by $0=\omega_{0}<\omega_{1}<\ldots<\omega_{J}=\omega$. The function $\varepsilon$ is positive and constant on each subinterval: $\varepsilon=\varepsilon_{j}$ for $\theta \in\left(\omega_{j-1}, \omega_{j}\right)$.

The function $u$ is homogeneous in $\Gamma$ and satisfies

$$
\Delta_{\varepsilon}^{\text {Dir }} u=0 \quad\left[\text { or } \Delta_{\varepsilon}^{\text {Neu }} u=0\right] .
$$

Thus $u(r, \theta)=r^{\lambda} v(\theta)$ with $\lambda>0$ and $v$ is a linear combination of $\sin \lambda \theta$ and $\cos \lambda \theta$ in each $\left(\omega_{j-1}, \omega_{j}\right)$ satisfying the boundary conditions

$$
v(0)=v(\omega)=0 \quad\left[\text { or } v^{\prime}(0)=v^{\prime}(\omega)=0\right]
$$

and the transmission conditions

$$
[v]=0 \quad \text { and } \quad\left[\varepsilon v^{\prime}\right]=0 \quad \text { at } \theta=\omega_{j}
$$


Under these conditions, we have the following result:

\section{Theorem 8.1.}

(i) (External edge)

If $\omega<2 \pi$ and $J=2$, then $\lambda>\pi / 2 \omega$.

(ii) (Internal edge)

If $\omega=2 \pi$ and $J=2$, then $\lambda>1 / 2$;

If $\omega=2 \pi$ and $J=3$, then $\lambda>1 / 4$.

(iii) If $\omega<2 \pi$ and $J \geq 3$ or if $\omega=2 \pi$ and $J \geq 4$, then for any $\lambda_{0}>0$ there exist $\varepsilon_{1}, \ldots, \varepsilon_{J}$ and a function $u \neq 0$ with $0<\lambda<\lambda_{0}$.

Proof. (i) Consider first the case of Dirichlet conditions: the function $v$ is continuous on $[0, \omega]$, piecewise analytic, vanishes at 0 and $\omega$, and its derivative satisfies $\varepsilon_{1} v^{\prime}\left(\omega_{1}^{-}\right)=\varepsilon_{2} v^{\prime}\left(\omega_{1}^{+}\right)$. One can assume that $v$ has a positive maximum in $\omega^{*} \in(0, \omega)$. It follows that $v^{\prime}\left(\omega^{*}\right)=0$, even if $\omega^{*}=\omega_{1}$, because $v^{\prime}$ does not change its sign there. In one of the two sectors $\left(0, \omega^{*}\right)$ (if $\omega^{*} \leq \omega_{1}$ ) or $\left(\omega^{*}, \omega\right)$ (if $\omega^{*}>\omega_{1}$ ), the function $u$ therefore satisfies a mixed Dirichlet-Neumann problem without interface, for which one knows the lowest singularity exponent $\pi / 2 \omega^{*}$ or $\pi / 2\left(\omega-\omega^{*}\right)$. Thus

$$
\lambda \geq \frac{\pi}{2 \omega^{*}} \geq \frac{\pi}{2 \omega_{1}}>\frac{\pi}{2 \omega} \quad \text { or } \quad \lambda \geq \frac{\pi}{2\left(\omega-\omega^{*}\right)} \geq \frac{\pi}{2\left(\omega-\omega_{1}\right)}>\frac{\pi}{2 \omega} .
$$

For exterior Neumann conditions, we have $v^{\prime}(0)=v^{\prime}(\omega)=0$. Since $v$ is an eigenfunction of the Laplace-Beltrami Neumann problem, it is orthogonal to constants:

$$
\int_{0}^{\omega} v(\theta) \varepsilon(\theta) \mathrm{d} \theta=0
$$

As $\varepsilon$ is positive, $v$ has at least one zero: $v\left(\omega^{*}\right)=0$. Once again, on either $\left(0, \omega^{*}\right)$ or $\left(\omega^{*}, \omega\right)$, we obtain a mixed Dirichlet-Neumann problem and the estimate

$$
\lambda \geq \min \left\{\frac{\pi}{2 \omega_{1}}, \frac{\pi}{2\left(\omega-\omega_{1}\right)}\right\}>\frac{\pi}{2 \omega}
$$

(ii) If $\omega=2 \pi$, we can again use that $v$ is orthogonal to constant functions:

$\int_{0}^{2 \pi} v \varepsilon \mathrm{d} \theta=0$. This time, we conclude that $v$ has at least two distinct zeros

$0 \leq \omega^{*}<\omega^{* *}<2 \pi ; v\left(\omega^{*}\right)=v\left(\omega^{* *}\right)=0$. In the two sectors

$$
\Gamma^{*}=\left\{(r, \theta) \mid \omega^{*}<\theta<\omega^{* *}\right\} \quad \text { and } \quad \Gamma^{* *}=\left\{(r, \theta) \mid \omega^{* *}<\theta<2 \pi+\omega^{*}\right\}
$$

our function $u$ solves therefore the transmission problem with exterior Dirichlet conditions, and we are back to case (i).

If $J=2$, we can either argue that one of $\Gamma^{*}$ or $\Gamma^{* *}$ is convex, or that one of the two sectors contains only one material. Both arguments give the result $\lambda>1 / 2$.

If $J=3$, then one of the two sectors contains at most two materials, thus from (i) follows $\lambda>1 / 4$.

(iii) For the case $J=4$, we give the following explicit example: let

$$
G_{1}=\left(-\frac{\pi}{4}, \frac{\pi}{4}\right), \quad G_{2}=\left(\frac{\pi}{4}, \frac{3 \pi}{4}\right), \quad G_{3}=\left(\frac{3 \pi}{4}, \frac{5 \pi}{4}\right), \quad G_{4}=\left(-\frac{3 \pi}{4},-\frac{\pi}{4}\right) .
$$

and

$$
\varepsilon_{1}=\varepsilon_{3}=h \quad \text { and } \quad \varepsilon_{2}=\varepsilon_{4}=1
$$


Let $v$ be defined as $\sin \lambda \theta$ in $G_{1}, \eta \cos \lambda\left(\frac{\pi}{2}-\theta\right)$ in $G_{2}, \sin \lambda(\pi-\theta)$ in $G_{3},-\eta \cos \lambda\left(\frac{\pi}{2}+\theta\right)$ in $G_{4}$. Then $u(r, \theta)=r^{\lambda} v(\theta)$ is a singular function for our transmission problem if and only if

$$
\eta=\tan \frac{\lambda \pi}{4} \quad \text { and } \quad h=\eta^{2} .
$$

We see that $\lambda \rightarrow 0$ as $h \rightarrow 0$.

Since $u$ satisfies Dirichlet conditions at $\theta=0$ and $\theta=\pi$, the same example solves a 3 -material problem with exterior Dirichlet conditions.

This example can be easily adapted to more general geometries.

Remark 8.2. In the example of the proof of (iii), we have a three-material Dirichlet problem with a smooth exterior boundary. If we assume homogeneous magnetic properties, we have no type 1 edge singularity for the magnetic field there. The type 2 edge singularity has only regularity $H^{1+\delta}$ for $\delta<\lambda_{0}$.

\section{REFERENCES}

[1] C. Amrouche, C. Bernardi, M. Dauge and V. Girault, Vector potentials in three-dimensional nonsmooth domains. Math. Methods Appl. Sci. 21 (1998) 823-864.

[2] F. Assous, P. Ciarlet and E. Sonnendrücker, Résolution des équations de Maxwell dans un domaine avec un coin rentrant. C.R. Acad. Sci. Paris, Ser. I 323 (1996) 203-208.

[3] M. Birman and M. Solomyak, $L^{2}$-theory of the Maxwell operator in arbitrary domains. Russ. Math. Surv. 42 (1987) $75-96$.

[4] M. Birman and M. Solomyak, On the main singularities of the electric component of the electro-magnetic field in regions with screens. St. Petersbg. Math. J. 5 (1993) 125-139.

[5] A. Bonnet-BenDhia, C. Hazard and S. Lohrengel, A singular field method for the solution of Maxwell's equations in polyhedral domains. SIAM J. Appl. Math. (to appear).

[6] M. Costabel, A remark on the regularity of solutions of Maxwell's equations on Lipschitz domains. Math. Methods Appl. Sci. 12 (1990) 365-368.

[7] M. Costabel, A coercive bilinear form for Maxwell's equations. J. Math. Anal. Appl. 157 (1991) 527-541.

[8] M. Costabel and M. Dauge, Singularities of electromagnetic fields in polyhedral domains. Arch. Rational Mech. Anal. (to appear).

[9] M. Dauge, Elliptic Boundary Value Problems in Corner Domains - Smoothness and Asymptotics of Solutions. Lect. Notes Math. Vol. 1341. Springer-Verlag, Berlin (1988).

[10] M. Dobrowolski, Numerical approximation of elliptic interface and corner problems. Habilitationsschrift, Bonn (1981).

[11] P. Fernandes and G. Gilardi, Magnetostatic and electrostatic problems in inhomogeneous anisotropic media with irregular boundary and mixed boundary conditions. Math. Models Methods Appl. Sci. 7 (1997) 957-991.

[12] N. Filonov, Système de Maxwell dans des domaines singuliers. Ph.D. thesis, University of Bordeaux 1, France (1996).

[13] V.A. Kondrat'ev, Boundary-value problems for elliptic equations in domains with conical or angular points. Trans. Moscow Math. Soc. 16 (1967) 227-313.

[14] T. Kühn, Die Regularität der Lösungen von Interface-Problemen in Gebieten mit singulären Punkten. Ph.D. thesis, University of Rostock (1992).

[15] D. Leguillon and E. Sanchez-Palencia, Computation of singular solutions in elliptic problems and elasticity. RMA 5. Masson, Paris (1987).

[16] K. Lemrabet, Régularité de la solution d'un problème de transmission. J. Math. Pures Appl. 56 (1977) 1-38.

[17] M. Moussaoui, Espaces H(div, rot, $\Omega$ ) dans un polygone plan. C.R. Acad. Sci. Paris, Ser. I 322 (1996) $225-229$.

[18] S. Nicaise, Polygonal interface problems. Methoden und Verfahren der Mathematischen Physik, 39. Verlag Peter D. Lang, Frankfurt-am-Main (1993).

[19] S. Nicaise and A.M. Sändig, General interface problems I/II. Math. Methods Appl. Sci. 17 (1994) 395-450.

[20] J. Saranen, On an inequality of Friedrichs. Math. Scand. 51 (1982) 310-322. 